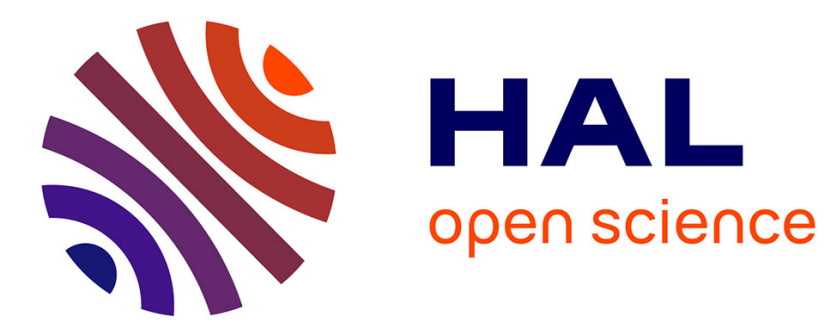

\title{
Optimizing subscriber migrations for a telecommunication operator in uncertain context
}

Adrien Cambier, Matthieu Chardy, Rosa Figueiredo, Adam Ouorou, Michael Poss

\section{- To cite this version:}

Adrien Cambier, Matthieu Chardy, Rosa Figueiredo, Adam Ouorou, Michael Poss. Optimizing subscriber migrations for a telecommunication operator in uncertain context. European Journal of Operational Research, 2022, 298 (1), pp.308-321. 10.1016/j.ejor.2021.06.032 . hal-02893167v2

\section{HAL Id: hal-02893167 \\ https://hal.science/hal-02893167v2}

Submitted on 15 Jul 2021

HAL is a multi-disciplinary open access archive for the deposit and dissemination of scientific research documents, whether they are published or not. The documents may come from teaching and research institutions in France or abroad, or from public or private research centers.
L'archive ouverte pluridisciplinaire HAL, est destinée au dépôt et à la diffusion de documents scientifiques de niveau recherche, publiés ou non, émanant des établissements d'enseignement et de recherche français ou étrangers, des laboratoires publics ou privés. 


\title{
Optimizing subscriber migrations for a telecommunication operator in uncertain context
}

\author{
Adrien Cambiera,b,*, Matthieu Chardy ${ }^{\mathrm{a}}$, Rosa Figueiredo ${ }^{\mathrm{b}}$, Adam Ouorou $^{\mathrm{a}}$, Michael Poss $^{\mathrm{c}}$ \\ ${ }^{a}$ Orange Labs, 44 avenue de la République, 92320 Châtillon, France. \\ ${ }^{b}$ LIA, Avignon Université, Avignon, France. \\ ${ }^{c}$ UMR CNRS 5506 LIRMM, Université de Montpellier, Montpellier, France.
}

\begin{abstract}
We consider a telecommunications company expanding its network capacity to face an increasing demand. The company can also invest in marketing to incentivize clients to shift to more recent technologies, hopefully leading to cheaper overall costs. To model the effect of marketing campaigns, previous works have relied on the Bass model. Since that model only provides a rough approximation of the actual shifting mechanism, the purpose of this work is to consider uncertainty in the shifting mechanism through the lens of robust optimization. We thus assume that the (discrete) shifting function can take any value in a given polytope and wish to optimize against the worst-case realization. The resulting robust optimization problem possesses integer recourse variables and non-linear dependencies on the uncertain parameters. We address these difficulties as follows. First, the integer recourse is tackled heuristically through a piece-wise constant policy dictated by a prior partition of the uncertainty polytope. Second, the non-linearities are handled by a careful analysis of the dominating scenarios. The scalability and economical relevance of our models are assessed through numerical experiments performed on realistic instances. In particular, we choose one of these instances to perform a case study with simulations illustrating the possible benefit of using robust optimization.

Keywords: OR in telecommunications, Capacity Expansion, Bass model, Mixed-Integer Linear Programming, Robust Optimization
\end{abstract}

\footnotetext{
${ }^{*}$ Corresponding author

Email address: adrien.cambier@orange.com (Adrien Cambier)
} 


\section{Introduction and problem description}

New bandwidth-consuming usages and the increase in the number of users induce an exponential growth of mobile traffic (CISCO, 2017). Facing this traffic growth, telecommunications companies are hence pushed to expand their network through important investments (several billion $€$ to improve the mobile network in the last six years, see Orange (2018)). This network design problem has two specific features. First, a fast roll-out of mobile network generations and a highly competitive environment that encourages operators to invest in the newest technology available. Second, telecommunications companies are often both infrastructure operators, planning their network expansion, and service providers, designing the offers proposed to the subscribers. These two features impact the investment decisions of the operator.

Through marketing investments, the operator can control the demand on its different technologies and avoid over-dimensioning, hence reducing its investments in network design. Reversely, the efficiency of such investments over a given year is also dependent on the network deployment performed in the previous years: subscribers will easily accept to shift towards the newest technology if it benefits from a high level of coverage, thanks to previous investments in the deployment of that technology. Exploring the trade-off between network and subscribers dynamic can be financially more interesting than a separate optimization of the two problems. Moreover, an operator fixes strategic guidelines on its network to remain competitive. Some of these guidelines, for example throughput, depend on both network and subscriber performances. Investments in subscribers and networks should hence be jointly optimized over the whole time-horizon of strategic planning, which is typically 5 years for a telecommunication operator.

This enlightens that subscriber and network dynamics are intertwined, motivating the introduction by Cambier et al. (2020) of an optimization problem that considers jointly the two aspects. In Cambier et al. (2020) the problem is formulated as a mixed-integer linear program (MILP). This MILP is obtained by considering discrete subsidies that represent different possible marketing savings on a new phone required to access the new service. To model how the subscribers react to such subsidies, they use a discretized shifting function (providing the number of subscribers deciding to shift to the newest generation) that is inspired by the well-known Bass model (see Bass (1969); Norton and Bass (1987); Bass (2004)). The authors of Cambier et al. (2020) tackle a naive deterministic approximation where the subsidy offered and the network deployment directly 
provide the shifting value. In practice however, the actual shifting value is typically not known with precision, as many internal and external factors intervene in operator marketing, perturbing the value dictated by the Bass model.

We model the shifting function uncertainty via a known polyhedral set, leading to a robust optimization problem. Robust optimization is an efficient framework to handle uncertainty in (mixed-integer) linear optimization problems, see Ben-Tal et al. (2009) and Gabrel et al. (2014), among others. The framework can, in theory, address a wide variety of optimization problems, including discrete variables (Buchheim and Kurtz, 2018), non-linear constraints (Ben-Tal et al., 2015), and the multi-stage setting where some of the decision variables can adjust their value to the realization of the uncertain parameters (see Delage and Iancu (2015) and İhsan Yanıkoğlu et al. (2019)). In practice, however, the dimensions of the problems one can expect to solve, and the optimality guarantees of the resulting algorithms, strongly depend on the problem characteristics and on the difficulty of the underlying nominal optimization problem. Here one must bear in mind that the underlying model considered, which was studied in Cambier et al. (2020), is already a difficult MILP that can be solved exactly only for moderate-size instances. This means that the robust counterpart we seek should not be much harder than its nominal variant.

The specific application considered in this paper features two specific difficulties: some of the integer variables are adjustable, and the constraints of the problem have non-linear dependencies on the shifting function. While both specificities have been addressed in the recent robust optimization literature, they are often addressed through heuristic solution procedures. On the one hand, integer adjustable variables are typically tackled (approximately) by partitioning the uncertainty polytope (Bertsimas and Caramanis, 2010; Bertsimas and Dunning, 2016; Postek and den Hertog, 2016; Ben-Ameur and Zotkiewicz, 2011) or by introducing complex decision rules (Bertsimas and Georghiou, 2018). On the other hand, robust optimization with non-linear dependency on the uncertainty parameters can, in some cases, be reformulated efficiently, for instance, when these dependencies are concave functions (Ben-Tal et al., 2015). The non-linear dependencies considered herein will be modeled by (non-concave) bilinear functions for which reformulations exist only in very particular cases, e.g., when the uncertainty polytope is an ellipsoid (Ben-Tal et al., 2002) or if the function is the product of two affine functions (Pessoa and Poss, 2015).

In this paper, we address the adjustable variables through uncertainty partitioning, while the 
non-linearities are handled by underlining dominances of the uncertainty polytope. Specifically, our contribution is three-fold. First, we provide two formulations for the two-period deterministic problem: one comes directly from Cambier et al. (2020) and the other one is a reformulation for the two period-case which will be more amenable to the derivation of the robust counterpart. These formulations are strengthened with Reformulation Linearization Techniques (RLT) inequalities (see Sherali and Adams (1998)). Second, we provide two robust counterparts (static and adjustable) of the previous model, as well as a solving method. While our static model is valid for any uncertainty polytope, our adjustable piece-wise constant approach critically requires to use a box as uncertainty polytope. This method analyzes the structure of the problem to reformulate it as a linear mixed-integer program with a finite number of constraints and variables. Third, through numerical experiments on realistic instances, we provide insights for the operator on the effects of uncertainty, and the importance to adapt her decisions to the shifting function outcome in the first-stage.

The remainder of this article is organized as follows. Section 2 presents the two mixed-integer formulations for the deterministic framework. Section 3 introduces the static and adjustable robust counterparts. A case study assessing the performances of the static and adjustable models on an operational context is provided in Section 4. In Section 5, we assess the scalability of our different formulations. Concluding remarks are given in Section 6 .

\section{Deterministic model and formulations}

In this section, we present two formulations for the two-periods deterministic framework. Section 2.1 introduces the notations used and formally describes the problem. Section 2.2 presents the formulation from Cambier et al. (2020). A reformulation is provided in Section 2.3. This reformulation is linearized and strengthened in Section 2.4.

\subsection{Problem description}

\subsubsection{Network dynamic}

In this work, we focus on a framework with two network technologies (denoted by $g \in \mathcal{G}=$ $\{C G, N G\}$ ), the current one $C G$ and the newest one $N G$ that the telecommunication operator aims to deploy. The time horizon is discretized in two equally-sized time periods, denoted by $t \in \mathcal{T}=$ $\{1,2\}$, adding " 0 " to denote the beginning of the time-horizon. We consider a set $\mathcal{S}=\left\{1, \ldots, N_{S}\right\}$ 
of $N_{S}$ existing telecommunication sites. At the beginning of the time-horizon, all sites are equipped with $C G$ technology, while $N G$ is deployed on some sites and can be deployed on the other ones. These sites have an initial capacity (discretized in numbers of modules) which can be increased by adding new modules. We also consider that we know an equivalent number of subscribers associated with each site $s \in \mathcal{S}$. A technical compatibility rule imposes that $C G$ subscribers can only be served by $C G$ technology while $N G$ subscribers can be served by both technologies. Furthermore, the load-balancing rule decided by the operator states that $N G$ subscribers have to be served by $N G$ technology if $N G$ is deployed. The capacity of each site and each technology has to be sufficient for handling traffic $95 \%$ of the time.

\subsubsection{Shifting subscribers}

We also assume that the upgrade mechanism modeling subscribers reaction for shifting to $N G$ technology depends on two parameters:

- The value of the subsidy denoted by $\sigma$.

- A discrete indicator of the level of coverage of $N G$ technology denoted by $c$.

The set of possible subsidy values is denoted by $\mathcal{K}$. We model the level of coverage by partitioning the interval $[0,1]$ into $C$ smaller intervals indexed by set $\mathcal{C}=\{1, \ldots, C\}$. Hence $c \in \mathcal{C}$ indicates the interval (coverage range) to which belongs the sites coverage (percentage of sites equipped with $N G$ at the beginning of the time period).

We hence denote the percentage of subscribers who shift when being proposed a subsidy $\sigma \in \mathcal{K}$ under a level of coverage $c \in \mathcal{C}$ by $f_{\sigma, c}$. This parameter should respect the two following rules. First, for a given coverage $\tilde{\sigma} \in \mathcal{K}$, the function $c \rightarrow f_{\tilde{\sigma}, c}$ is non-decreasing (since the higher the coverage is, the higher the subscribers reaction is). Second, for a given coverage $\tilde{c} \in \mathcal{C}$, the function $\sigma \rightarrow f_{\sigma, \tilde{c}}$ is S-shaped. Such function is used for modeling two phenomena appearing in the arrival of a new technology:

- the beginning of the function is convex: it models that a threshold in subsidy amount has to be reach for having an important increase in the reaction. This threshold corresponds to the minimum subsidy proposal required to convince more subscribers than only the very technology-friendly ones. 
- the end of the function is concave: it models laggards subscribers, which are the subscribers who do not want to shift and will not shift, whatever the subdisdy proposal is - see Bass (1969) for more details.

\subsubsection{Strategic guidelines}

We further consider two strategic guidelines that can be fixed by a telecommunication regulator or the operator: the quality of experience (percentage of $N G$ subscribers served by $N G$ technology) and the sites coverage, both taken at the end of the time-horizon.

\subsubsection{Problem statement}

All in all, the Mobile Master Plan (MMP) problem for these two technologies consists in finding the subsidies decisions (amount of subsidy given at each period) and networks decisions (installing $N G$ technology and adding modules for both technologies) for each site, while satisfying loadbalancing and capacity constraints at each time period, and the two strategic guidelines at the end of the time horizon.

\subsubsection{Sum-up of parameters and variables used}

Parameters and variables used throughout this paper are provided respectively in Tables 1 and 2 . 


\begin{tabular}{|c|c|}
\hline \multicolumn{2}{|c|}{ Parameters: } \\
\hline$C A_{N G}$ & cost of adding $N G$ technology on each site \\
\hline$C M_{g}$ & cost of adding a module of technology $g \in \mathcal{G}$ on each site \\
\hline$M_{s, g}^{0}$ & initial number of modules of technology $g \in \mathcal{G}$ on site $s \in \mathcal{S}$ \\
\hline $\bar{M}_{g}$ & technical upper bound on the number of modules of technology $g \in \mathcal{G}$ \\
\hline$Z_{s, N G}^{0}$ & initial presence (yes/no) of $N G$ technology on site $s \in \mathcal{S}$ \\
\hline$U_{s, g}^{0}$ & initial number of subscribers on site $s \in \mathcal{S}$ to technology $g \in \mathcal{G}$ \\
\hline$D_{g}^{t}$ & unitary demand of a subscriber served by technology $g \in \mathcal{G}$ at time period $t \in \mathcal{T}$ \\
\hline$C A P_{g}$ & capacity of each additional module of technology $g \in \mathcal{G}$ \\
\hline$f_{\sigma, c}$ & reaction to the subsidy offered $\sigma \in \mathcal{K}$ under range of coverage interval $c \in \mathcal{C}$ \\
\hline$L_{c}$ & lower bound of coverage range indexed by $c \in \mathcal{C}$ \\
\hline$U_{c}$ & upper bound of coverage range indexed by $c \in \mathcal{C}$ \\
\hline $\bar{U}_{s, g}^{t}$ & $\begin{array}{l}\text { upper bound on the total number of subscribers to technology } g \in \mathcal{G} \text { on site } s \in \mathcal{S} \\
\text { at the end of time period } t \in \mathcal{T}\end{array}$ \\
\hline$\alpha^{0}$ & sites coverage at the beginning of the time horizon \\
\hline$\underline{\alpha}$ & threshold for the coverage (strategic guideline) \\
\hline$\underline{Q} \underline{\underline{B}}$ & threshold for the quality of service (strategic guideline) \\
\hline
\end{tabular}

Table 1: Model parameters 




Table 2: Model variables 


\subsection{Mixed-integer linear formulation for the two-periods deterministic problem}

The MMP problem is modeled in Cambier et al. (2020) as follows:

$$
\begin{aligned}
\min & \sum_{t \in \mathcal{T}} \sum_{\sigma \in \mathcal{K}} \sum_{c \in \mathcal{C}} \sum_{s \in \mathcal{S}} \sigma f_{\sigma, c} \delta_{\sigma}^{t} \gamma_{c}^{t} u_{s, C G}^{t-1}+\sum_{s \in \mathcal{S}} \sum_{g \in \mathcal{G}} C M_{g}\left(m_{s, g}^{2}-M_{s, g}^{0}\right) \\
& +\sum_{s \in \mathcal{S}} C A_{N G}\left(z_{s, N G}^{2}-Z_{s, N G}^{0}\right)
\end{aligned}
$$




$$
\begin{array}{ll}
z_{s, N G}^{0}=Z_{s, N G}^{0} & \forall s \in \mathcal{S}, \\
m_{s, g}^{t} \in \mathbb{N} & \forall s \in \mathcal{S}, \forall t \in \mathcal{T} \cup\{0\}, \forall g \in \mathcal{G}, \\
z_{s, N G}^{t} \in\{0,1\} & \forall s \in \mathcal{S}, \forall t \in \mathcal{T} \cup\{0\}, \\
u_{s, o}^{t} \geq 0 & \forall s \in \mathcal{S}, \forall t \in \mathcal{T} \cup\{0\}, \forall o \in \mathcal{G}, \\
u_{s, o, g}^{t} \geq 0 & \forall s \in \mathcal{S}, \forall t \in \mathcal{T}, \forall o, g \in \mathcal{G}^{2}, \\
\gamma_{c}^{t} \in\{0,1\} & \forall t \in \mathcal{T}, \forall c \in \mathcal{C}, \\
\delta_{\sigma}^{t} \in\{0,1\} & \forall t \in \mathcal{T}, \forall \sigma \in \mathcal{K} .
\end{array}
$$

We denote this formulation by $\mathcal{M}^{\text {det }}$. The objective function (1) minimizes both subscribers' migration costs and network investments. The first term stands for the cost of offering subsidies to the subscriber, the second term for the network modules cost, and the third term for the deployment of the newest technology $N G$.

Constraints (2)-(4) enforce the network dynamic rules ensuring the number of modules to be non-decreasing and imposing technical upper bounds on numbers of modules. Constraints (5)-(8) refer to the network dimensioning constraints, ensuring capacity constraints and load-balancing rules, making hence the link between the network dynamic and the subscriber dynamic. Constraints (9) and (10) stand for the subscriber dynamic constraints. They compute the total number of subscribers to $C G$ and $N G$ technologies at each site and each time period in terms of the number of subscribers to both technologies in the previous period and of the percentage of former $C G$ subscribers who decide to shift to $N G$ technology. Constraints (11) and (12) ensure the model strategic guidelines and refer to the end of the time horizon. Constraints (13) ensure that one and only one subsidy from the set $\mathcal{K}$ is offered at each time period. Constraints (14)-(16) set the range of sites coverage for each time period according to the sites coverage. Constraints (17) define the sites coverage. Constraints (18)-(20) refer to the initial conditions and constraints while constraints (21)-(26) define the domain of all variables in the formulation.

To linearize this formulation, we introduce variables:

$$
\pi_{\sigma, c, s}^{t}=\delta_{\sigma}^{t} \gamma_{c}^{t} u_{s, C G}^{t-1}, \forall t \in \mathcal{T}, \forall s \in \mathcal{S}, \forall \sigma \in \mathcal{K}, \forall c \in \mathcal{C}
$$

and use the classical linearization of the product of a binary variable by a continuous one. Details on the linearization are given in Cambier et al. (2020). 


\subsection{Reformulation}

As mentioned in the introduction, the goal of this paper is to study a robust counterpart of $\mathcal{M}^{\text {det }}$ wherein $f$ is uncertain and $\delta$ is adjustable, meaning its value for period $t$ depends on the realizations of $f$ in the previous periods. As a consequence of constraints (9) and (10), variables $u$ have to be adjustable as well. Therefore, a robust counterpart of $\mathcal{M}^{\text {det }}$ would involve products of adjustable real variables with uncertain parameters, for which affine decision rules could not be used. To overcome that difficulty, we consider in this section a reformulation that uses the recursive structure defined by constraints (9) and (10) to expresses variables $u$ directly in term of variables $\delta$, $\gamma$ and of the function $f$. This reformulation will be more amenable to obtain a robust counterpart in our two-periods framework.

Proposition 2.1. The following non-linear equalities are valid expressions for the number of subscribers on each site $s \in \mathcal{S}$ and each time-period $t \in \mathcal{T}$ in $\mathcal{M}^{\text {det }}$

$$
u_{s, C G}^{t}=U_{s, C G}^{0} \prod_{i=1}^{t}\left(1-\sum_{\sigma \in \mathcal{K}} \sum_{c \in \mathcal{C}} f_{\sigma, c} \delta_{\sigma}^{i} \gamma_{c}^{i}\right)
$$

and

$$
u_{s, N G}^{t}=U_{s, C G}^{0}+U_{s, N G}^{0}-u_{s, C G}^{t}=U_{s, N G}^{0}+U_{s, C G}^{0}\left[1-\prod_{i=1}^{t}\left(1-\sum_{\sigma \in \mathcal{K}} \sum_{c \in \mathcal{C}} f_{\sigma, c} \delta_{\sigma}^{i} \gamma_{c}^{i}\right)\right]
$$

Proof. The expression for $u_{s, C G}^{t}$ is obtained by applying recursively over the time-horizon constraints (9) from $\mathcal{M}^{\text {det }}$. The expression for $u_{s, N G}^{t}$ is obtained by summing constraints (9) and (10) which gives $u_{s, C G}^{t}+u_{s, N G}^{t}=u_{s, C G}^{t-1}+u_{s, N G}^{t-1}$ and hence recursively $u_{s, C G}^{t}+u_{s, N G}^{t}=U_{s, C G}^{0}+U_{s, N G}^{0}$.

These expressions allow to replace continuous variables $u_{s, N G}^{t}$ and $u_{s, C G}^{t}$ by expressions that depend only on variables $\delta$ and $\gamma$. When considering $|\mathcal{T}|>2$ periods, these new expressions contain a high number of non-linearities (products of $\delta$ ) which require each a linearization variable: hence

$$
N V(|\mathcal{T}|)=\sum_{l=2}^{|\mathcal{T}|} \frac{|\mathcal{T}| !}{(|\mathcal{T}|-l) ! l !}|\mathcal{K}|^{l}=(|\mathcal{K}|+1)^{|\mathcal{T}|}-1-|\mathcal{T}||\mathcal{K}|
$$

additional variables are required. When focusing on a two periods framework $(T=2)$, the nonlinear terms are direct products of variables $\delta^{1}$ and $\delta^{2}$. We can hence linearize our formulation by adding only $|K|^{2}$ variables and $3|K|^{2}$ constraints (see Fortet (1960)). We observe that variables $\gamma^{1}$ are not needed as the coverage range in the first period depends on the initial percentage of $N G$ 
sites, and is hence already known.

Proposition 2.2. Constraints (5) and (6) can be replaced with the following non-linear equations in formulation $\mathcal{M}^{\text {det }}$, for each site $s \in \mathcal{S}$ and each time-period $t \in \mathcal{T}$ :

$$
\begin{array}{r}
u_{s, N G, N G}^{t}=u_{s, N G}^{t} z_{s, N G}^{t} \\
u_{s, N G, C G}^{t}=u_{s, N G}^{t}\left(1-z_{s, N G}^{t}\right)
\end{array}
$$

Proof. First, let us assume that equations (27) and (28) are satisfied. For each site $s \in \mathcal{S}$, by summing (27) and (28), we obtain that (5) is satisfied. Clearly, equation (6) is implied by (28).

Reciprocally, let us assume that constraints (5) and (6) are both satisfied. Two cases can happen. If $N G$ technology is installed $\left(z_{s, N G}^{t}=1\right)$ : constraints (6) induce $u_{s, N G, C G}^{t}=0$ and then $u_{s, N G}^{t}=u_{s, N G, N G}^{t}$ so that equations (27) and (28) are satisfied. If $N G$ technology is not installed $\left(z_{s, N G}^{t}=0\right)$, we have $m_{s, N G}^{t}=0$ (see constraints $\left.(3)\right)$ and $u_{s, N G, N G}^{t}=0$ (see constraints (8)) so that equations (27) and (28) are satisfied.

From Propositions 2.1 and 2.2, we obtain that continuous variables $u$ can be expressed with the following equations depending on $\delta, \gamma$ and $f$, for each site $s \in \mathcal{S}$ and each time-period $t \in \mathcal{T}$ :

$$
\begin{array}{ll}
u_{s, N G, N G}^{t}=\left(U_{s, N G}^{0}+U_{s, C G}^{0}\left[1-\prod_{i=1}^{t}\left(1-\sum_{\sigma \in \mathcal{K}} \sum_{c \in \mathcal{C}} f_{\sigma, c} \delta_{\sigma}^{i} \gamma_{c}^{i}\right)\right]\right) z_{s, N G}^{t} & \\
u_{s, C G}^{t}+u_{s, N G, C G}^{t}=u_{s, N G}^{t}+u_{s, C G}^{t}=U_{s, N G}^{0}+U_{s, C G}^{0} & \text { if } z_{s, N G}^{t}=0, \\
u_{s, C G}^{t}+u_{s, N G, C G}^{t}=u_{s, C G}^{t}=U_{s, C G}^{0} \prod_{i=1}^{t}\left(1-\sum_{\sigma \in \mathcal{K}} \sum_{c \in \mathcal{C}} f_{\sigma, c} \delta_{\sigma}^{i} \gamma_{c}^{i}\right) & \text { if } z_{s, N G}^{t}=1 .
\end{array}
$$

For simplifying the subsequent notations, we denote the total number of subscribers $\left(\sum_{s \in \mathcal{S}} U_{s, C G}^{0}+\right.$ $\left.U_{s, N G}^{0}\right)$ by $U^{t o t}$.

Consequently, formulation $\mathcal{M}^{\text {det }}$ with two generations and two periods can be reformulated without continuous variables, as follows:

$$
\min C_{s u b}+\sum_{s \in \mathcal{S}} \sum_{g \in \mathcal{G}} C M_{g}\left(m_{s, g}^{2}-M_{s, g}^{0}\right)+\sum_{s \in \mathcal{S}} C A_{N G}\left(z_{s, N G}^{2}-Z_{s, N G}^{0}\right)
$$




$$
\begin{aligned}
& \text { s.t. } \quad \sum_{\sigma^{1} \in \mathcal{K}} \sigma^{1} f_{\sigma^{1}, c^{1}} \delta_{\sigma^{1}}^{1}+\sum_{\sigma^{2} \in \mathcal{K}} \sum_{c^{2} \in \mathcal{C}} \sigma^{2} f_{\sigma^{2}, c^{2}} \delta_{\sigma^{2}}^{2} \gamma_{c^{2}}^{2}\left(1-\sum_{\sigma^{1} \in \mathcal{K}} f_{\sigma^{1}, c^{1}} \delta_{\sigma^{1}}^{1}\right) \leq \frac{C_{s u b}}{\sum_{s \in \mathcal{S}} U_{s, C G}^{0}} \\
& (2)-(4) \\
& D_{C G}^{1} C A P_{C G}\left(U_{s, C G}^{0}+U_{s, N G}^{0}\right)\left(1-z_{s, N G}^{1}\right) \leq m_{s, C G}^{1} \quad \forall s \in \mathcal{S}, \\
& \frac{D_{C G}^{1}}{C A P_{C G}} U_{s, C G}^{0}\left(1-\sum_{\sigma^{1} \in \mathcal{K}} f_{\sigma^{1}, c^{1}} \delta_{\sigma^{1}}^{1}\right) \leq m_{s, C G}^{1} \quad \forall s \in \mathcal{S}, \\
& \frac{D_{N G}^{1}}{C A P_{N G}}\left(U_{s, N G}^{0}+U_{s, C G}^{0} \sum_{\sigma^{1} \in \mathcal{K}} f_{\sigma^{1}, c^{1}} \delta_{\sigma^{1}}^{1}\right) z_{s, N G}^{1} \leq m_{s, N G}^{1} \quad \forall s \in \mathcal{S}, \\
& \frac{D_{C G}^{2}}{C A P_{C G}}\left(U_{s, C G}^{0}+U_{s, N G}^{0}\right)\left(1-z_{s, N G}^{2}\right) \leq m_{s, C G}^{2} \quad \forall s \in \mathcal{S}, \\
& \frac{D_{C G}^{2} U_{s, C G}^{0}}{C A P_{C G}}\left[1-\left(\sum_{\sigma^{1} \in \mathcal{K}} f_{\sigma^{1}, c^{1}} \delta_{\sigma^{1}}^{1}+\sum_{\sigma^{2} \in \mathcal{K}} \sum_{c^{2} \in \mathcal{C}} f_{\sigma^{2}, c^{2}} \delta_{\sigma^{2}}^{2} \gamma_{c^{2}}^{2}\left(1-\sum_{\sigma^{1} \in \mathcal{K}} f_{\sigma^{1}, c^{1}} \delta_{\sigma^{1}}^{1}\right)\right)\right] \leq m_{s, C G}^{2} \\
& \forall s \in \mathcal{S}, \\
& \frac{D_{N G}^{2}}{C A P_{N G}}\left[U_{s, N G}^{0}+U_{s, C G}^{0}\left(\sum_{\sigma^{1} \in \mathcal{K}} f_{\sigma^{1}, c^{1}} \delta_{\sigma^{1}}^{1}+\sum_{\sigma^{2} \in \mathcal{K}} \sum_{c^{2} \in \mathcal{C}} f_{\sigma^{2}, c^{2}} \delta_{\sigma^{2}}^{2} \gamma_{c^{2}}^{2}\left(1-\sum_{\sigma^{1} \in \mathcal{K}} f_{\sigma^{1}, c^{1}} \delta_{\sigma^{1}}^{1}\right)\right)\right] z_{s, N G}^{2} \leq m_{s, N G}^{2} \\
& \forall s \in \mathcal{S}, \quad(36) \\
& \sum_{s \in \mathcal{S}}\left[U_{s, N G}^{0}+U_{s, C G}^{0}\left(\sum_{\sigma^{1} \in \mathcal{K}} f_{\sigma^{1}, c^{1}} \delta_{\sigma^{1}}^{1}+\sum_{\sigma^{2} \in \mathcal{K}} \sum_{c^{2} \in \mathcal{C}} f_{\sigma^{2}, c^{2}} \delta_{\sigma^{2}}^{2} \gamma_{c^{2}}^{2}\left(1-\sum_{\sigma^{1} \in \mathcal{K}} f_{\sigma^{1}, c^{1}} \delta_{\sigma^{1}}^{1}\right)\right)\right] z_{s, N G}^{2} \geq \underline{Q o E}, U^{t o t} \\
& \text { (12) - (26) } \\
& C_{\text {sub }} \geq 0 .
\end{aligned}
$$

Constraint (30) enables to compute the subsidies cost $C_{\text {sub }}$ by using the expression from Proposition 2.1 for the number of $C G$ subscribers (note that variable $C_{s u b}$ will thanks to the minimization exactly be equal to the subsidies cost, but formulating with a $\leq$ sign is more amenable for writing the robust counterpart). Load-balancing constraints (5) and (6), capacity constraints (7) and (8), subscriber dynamic constraints (9) and (10) and $Q o E$ threshold constraint (11) from formulation $\mathcal{M}^{\text {det }}$ are replaced by set of constraints (31)-(37). Constraints (31) and (34) impose that all subscribers on a site (which is a constant) have to be served by $C G$ technology when $N G$ is not installed. Constraints (32) and (35) state that $C G$ subscribers have to be served by $C G$, which is dominated by previous constraints when $N G$ is not installed. Constraints (33) and (36) impose that when $N G$ is installed $N G$ subscribers have to be served by $N G$. The same formulas for obtaining the number of $N G$ subscribers are used in the QoE constraint (37). 


\subsection{Linearization and RLT cuts}

We discuss next how the model presented in Section 2.3 is linearized. Notice that all products appearing in this formulation are products of binary variables. We linearize the model presented in Section 2.3 by applying Fortet linearizations (see Fortet (1960)). For each $\sigma^{1}, \sigma^{2} \in \mathcal{K}^{2}, s \in \mathcal{S}$, $t \in \mathcal{T}$, we introduce the following new binary variables:

- $\chi_{\sigma, s}$ for linearizing $\delta_{\sigma}^{1} z_{s, N G}^{1}$ in constraints (33),

- $\pi_{\sigma, s}^{t}$ for linearizing $\delta_{\sigma}^{t} z_{s, N G}^{2}$ in constraints (36) and (37),

- $\eta_{\sigma^{1}, \sigma^{2}}$ for linearizing $\delta_{\sigma^{1}}^{1} \delta_{\sigma^{2}}^{2}$ in constraints $(30)$ and (35)-(37),

- $\zeta_{\sigma^{1}, \sigma^{2}, s}$ for linearizing $\delta_{\sigma^{1}}^{1} \delta_{\sigma^{2}}^{2} z_{s, N G}^{2}=\pi_{\sigma^{1}, s}^{1} \delta_{\sigma^{2}}^{2}=\pi_{\sigma^{2}, s}^{2} \delta_{\sigma^{1}}^{1}=z_{s, N G}^{2} \eta_{\sigma^{1}, \sigma^{2}}$ in constraints (36) and (37).

Remark 2.3. The variable $\delta^{2}$ and hence some of the variables above are also multiplied by $\gamma^{2}$. As in formulation $\mathcal{M}^{\text {det }}$, these products are not handled with Fortet linearizations, but by introducing variables $\delta_{\sigma, c}^{2}=\gamma_{c}^{2} \delta_{\sigma}^{2}$, for each $\sigma \in \mathcal{K}$ and each $c \in \mathcal{C}$, and by replacing $\gamma_{c}^{2}$ with $\sum_{\sigma \in \mathcal{K}} \delta_{\sigma, c}^{2}$ (term equal to 1 if coverage range is $c$ and 0 otherwise). We choose to keep $\gamma_{c}^{2} \delta_{\sigma}^{2}$ in the following for writing simplification.

We denote the corresponding linearized formulation by $\mathcal{M}^{\text {det,2p}}$. Now, we show how our formulation can be strengthened by applying Reformulation Linearization Techniques (RLT). Multiplying constraints (13) for $t=2$ by variable $z_{s, N G}^{1}$ for each site $s \in \mathcal{S}$,

$$
\sum_{\sigma \in \mathcal{K}} \chi_{\sigma, s}=z_{s, N G}^{1} \forall s \in \mathcal{S}
$$

Multiplying constraints (13) by variable $z_{s, N G}^{2}$ for each site $s \in \mathcal{S}$, we obtain:

$$
\sum_{\sigma \in \mathcal{K}} \pi_{\sigma, s}^{t}=z_{s, N G}^{2} \forall t \in \mathcal{T}, \forall s \in \mathcal{S}
$$

Multiplying constraints (13) for $t=2$ by variable $\delta_{\sigma^{1}}^{1}$ for each subsidy $\sigma^{1} \in \mathcal{K}$, we obtain:

$$
\sum_{\sigma^{2} \in \mathcal{K}} \eta_{\sigma^{1}, \sigma^{2}}=\delta_{\sigma^{1}}^{1} \forall \sigma^{1} \in \mathcal{K}
$$


Multiplying constraints (13) for $t=1$ by variable $\delta_{\sigma^{2}}^{2}$ for each subsidy $\sigma^{2} \in \mathcal{K}$, we obtain:

$$
\sum_{\sigma^{1} \in \mathcal{K}} \eta_{\sigma^{1}, \sigma^{2}}=\delta_{\sigma^{2}}^{2} \forall \sigma^{2} \in \mathcal{K}
$$

Summing constraints (41) (or (42)) on set $\mathcal{K}$, and applying constraints (13), we obtain:

$$
\sum_{\sigma^{1} \in \mathcal{K}} \sum_{\sigma^{2} \in \mathcal{K}} \eta_{\sigma^{1}, \sigma^{2}}=1
$$

Multiplying constraints (41) by variable $z_{s, N G}^{2}$ for each site $s \in \mathcal{S}$, we obtain:

$$
\sum_{\sigma^{2} \in \mathcal{K}} \zeta_{\sigma^{1}, \sigma^{2}, s}=\pi_{\sigma^{1}, s}^{1} \forall s \in \mathcal{S}, \forall \sigma^{1} \in \mathcal{K}
$$

Multiplying constraints (42) by variable $z_{s, N G}^{2}$ for each site $s \in \mathcal{S}$, we obtain:

$$
\sum_{\sigma^{1} \in \mathcal{K}} \zeta_{\sigma^{1}, \sigma^{2}, s}=\pi_{\sigma^{2}, s}^{2} \forall s \in \mathcal{S}, \forall \sigma^{2} \in \mathcal{K}
$$

Finally, multiplying constraints $(43)$ by variable $z_{s, N G}^{2}$ for each site $s \in \mathcal{S}$, we obtain:

$$
\sum_{\sigma^{1} \in \mathcal{K}} \sum_{\sigma^{2} \in \mathcal{K}} \zeta_{\sigma^{1}, \sigma^{2}}=z_{s, N G}^{2} \forall s \in \mathcal{S}
$$

Summarizing, formulation $\mathcal{M}^{\text {det,2p }}$ can be reinforced by adding constraints (40)-(46).

\section{Robust formulation}

In this section, we detail the static and adjustable robust counterparts for formulation $\mathcal{M}^{\text {det,2p}}$. The static counterpart is presented in Section 3.1. We show in Section 3.2 that this formulation can be formulated as a MILP that contains at most twice as many constraints as the nominal problem. Section 3.3 presents the adjustable counterpart. We mention that while the models from Sections 3.1 and 3.2 are valid for general uncertainty polytopes, those from Section 3.3 require the additional assumption that the uncertainty polytope be a box.

\subsection{Static robust counterpart}

We now consider that the shifting function is uncertain and belongs to the uncertainty polytope $\mathcal{F}$. Let us recall that the shifting function is a discrete function associating to each couple $(\sigma, c) \in$ 
$\mathcal{K} \times \mathcal{C}$ a reaction $f_{\sigma, c} \in[0,1]$. Therefore, the polytope $\mathcal{F}$ is a subset of the finite dimension space $[0,1]^{|\mathcal{K}| \cdot|\mathcal{C}|}$. The realized shifting functions may be different in each period. We thus denote by $f^{1} \in \mathcal{F}$ the shifting function realization in the first period and by $f^{2} \in \mathcal{F}$ the shifting function realization in the second period.

In a static framework, the decisions cannot be adapted to mitigate the effects of the uncertainty. Consequently, the static robust counterpart of formulation $\mathcal{M}^{\text {det,2p }}$ can be formulated as follows.

$$
\begin{aligned}
& \min C_{s u b}+\sum_{s \in \mathcal{S}} \sum_{g \in \mathcal{G}} C M_{g}\left(m_{s, g}^{2}-M_{s, g}^{0}\right)+\sum_{s \in \mathcal{S}} C A_{N G}\left(z_{s, N G}^{2}-Z_{s, N G}^{0}\right) \\
& \text { s.t. } \quad(2)-(4),(31),(34),(13)-(26) \\
& \sum_{\sigma^{1} \in \mathcal{K}} \sigma^{1} f_{\sigma^{1}, c^{1}}^{1} \delta_{\sigma^{1}}^{1}+\sum_{\sigma^{2} \in \mathcal{K}} \sum_{c^{2} \in \mathcal{C}} \sigma^{2} f_{\sigma^{2}, c^{2}}^{2} \delta_{\sigma^{2}}^{2} \gamma_{c^{2}}^{2}\left(1-\sum_{\sigma^{1} \in \mathcal{K}} f_{\sigma^{1}, c^{1}}^{1} \delta_{\sigma^{1}}^{1}\right) \leq \frac{C_{s u b}}{\sum_{s \in \mathcal{S}} U_{s, C G}^{0}} \\
& \forall f^{1} \in \mathcal{F}, \forall f^{2} \in \mathcal{F} \\
& D_{C G}^{1} U_{s, C G}^{0}\left(1-\sum_{\sigma \in \mathcal{K}} f_{\sigma, c^{1}}^{1} \delta_{\sigma}^{1}\right) \leq C A P_{C G} m_{s, C G}^{1} \quad \forall s \in \mathcal{S}, \forall f^{1} \in \mathcal{F}, \\
& D_{N G}^{1}\left(U_{s, N G}^{0}+U_{s, C G}^{0} \sum_{\sigma \in \mathcal{K}} f_{\sigma, c^{1}}^{1} \delta_{\sigma}^{1}\right) z_{s, N G}^{1} \leq C A P_{N G} m_{s, N G}^{1} \quad \forall s \in \mathcal{S}, \forall f^{1} \in \mathcal{F}, \\
& \frac{D_{C G}^{2} U_{s, C G}^{0}}{C A P_{C G}}\left[1-\left(\sum_{\sigma^{1} \in \mathcal{K}} f_{\sigma^{1}, c^{1}}^{1} \delta_{\sigma^{1}}^{1}+\sum_{\sigma^{2} \in \mathcal{K}} \sum_{c^{2} \in \mathcal{C}} f_{\sigma^{2}, c^{2}}^{2} \delta_{\sigma^{2}}^{2} \gamma_{c^{2}}^{2}\left(1-\sum_{\sigma^{1} \in \mathcal{K}} f_{\sigma^{1}, c^{1}}^{1} \delta_{\sigma^{1}}^{1}\right)\right)\right] \leq m_{s, C G}^{2} \\
& \forall s \in \mathcal{S}, \quad \forall f^{1} \in \mathcal{F}, \forall f^{2} \in \mathcal{F}, \\
& \frac{D_{N G}^{2}}{C A P_{N G}}\left[U_{s, N G}^{0}+U_{s, C G}^{0}\left(\sum_{\sigma^{1} \in \mathcal{K}} f_{\sigma^{1}, c^{1}}^{1} \delta_{\sigma^{1}}^{1}+\sum_{\sigma^{2} \in \mathcal{K}} \sum_{c^{2} \in \mathcal{C}} f_{\sigma^{2}, c^{2}}^{2} \delta_{\sigma^{2}}^{2} \gamma_{c^{2}}^{2}\left(1-\sum_{\sigma^{1} \in \mathcal{K}} f_{\sigma^{1}, c^{1}}^{1} \delta_{\sigma^{1}}^{1}\right)\right)\right] z_{s, N G}^{2} \leq m_{s, N G}^{2} \\
& \forall s \in \mathcal{S}, \forall f^{1} \in \mathcal{F}, \forall f^{2} \in \mathcal{F}, \\
& \sum_{s \in \mathcal{S}}\left[U_{s, N G}^{0}+U_{s, C G}^{0}\left(\sum_{\sigma^{1} \in \mathcal{K}} f_{\sigma^{1}, c^{1}}^{1} \delta_{\sigma^{1}}^{1}+\sum_{\sigma^{2} \in \mathcal{K}} \sum_{c^{2} \in \mathcal{C}} f_{\sigma^{2}, c^{2}}^{2} \delta_{\sigma^{2}}^{2} \gamma_{c^{2}}^{2}\left(1-\sum_{\sigma^{1} \in \mathcal{K}} f_{\sigma^{1}, c^{1}}^{1} \delta_{\sigma^{1}}^{1}\right)\right)\right] z_{s, N G}^{2} \geq \underline{Q o E} U^{t o t} \\
& \forall f^{1} \in \mathcal{F}, \forall f^{2} \in \mathcal{F} .
\end{aligned}
$$

Let us denote this formulation by $\mathcal{M}^{\text {stat }}$. Being a robust mixed-integer linear programming with polyhedral uncertainty, $\mathcal{M}^{\text {stat }}$ has an infinite number of constraints.

Remark 3.1. As for the deterministic model, formulation $\mathcal{M}^{\text {stat }}$ can be linearized and reinforced by constraints $(40)-(46)$.

\subsection{Constraints dominance}

We observe that the previous formulation contains constraints involving quadratic dependencies on $f$. This is the case for each constraint that involves the numbers of subscribers to each offer 
in the second period, i.e. constraints (48), (51), (52) and (53). Handling constraints with nonlinear dependencies of the uncertain parameters may not be easy in general. Fortunately, we show in this section that the specific structure of our constraints is simple enough to lead to a direct reformulation based on the dominance of set $\mathcal{F}$ by two vectors denoted by $\bar{f}$ and $\underline{f}$.

In what follows, let $\tilde{\delta}$ represent the value of $\delta$ in a feasible solution of $\mathcal{M}^{\text {stat }}$ and let $\tilde{\sigma}$ denote the subsidy offered in that solution. Similarly, let $\tilde{\gamma}^{2}$ represent the value of $\gamma^{2}$ in a feasible solution of $\mathcal{M}^{\text {stat }}$ and let $\tilde{c}^{2}$ denote the range of coverage in the second period in that solution. Our reformulation is based on the following properties satisfied by any feasible solution of formulation $\mathcal{M}^{\text {stat }}$ :

Proposition 3.2. The term $\sum_{\sigma \in \mathcal{K}} f_{\sigma, c^{1}}^{1} \tilde{\delta}_{\sigma}^{1}$ is equal to $f_{\tilde{\sigma}^{1}, c^{1}}^{1}$.

Proof. This comes directly from constraints (13), $\tilde{\sigma}^{1}$ being the index of the only non-zero component of $\tilde{\delta}^{1}$.

Proposition 3.3. The term $\sum_{\sigma \in \mathcal{K}} \sum_{c \in \mathcal{C}} f_{\sigma, c}^{2} \tilde{\delta}_{\sigma}^{2} \tilde{\gamma}_{c}^{2}$ is equal to $f_{\tilde{\sigma}^{2}, \tilde{c}^{2}}^{2}$.

Proof. This result comes from constraints (13) and (14), $\tilde{\sigma}^{2}$ being the index of the unique non-zero component of $\tilde{\delta}^{2}$ and $\tilde{c}^{2}$ being the index of the unique non-zero component of $\tilde{\gamma}^{2}$.

These two simple results enable us to replace all robust constraints by equivalent sets of constraints, involving at most the two aforementioned vectors $\underline{f}$ and $\bar{f}$, which are defined as follows for each subsidy offered $\sigma \in \mathcal{K}$ and each coverage range $c \in \mathcal{C}$ :

- the weakest possible reaction is $\underline{f}_{\sigma, c}=\min _{f \in \mathcal{F}} f_{\sigma, c}$,

- the strongest possible reaction is $\bar{f}_{\sigma, c}=\max _{f \in \mathcal{F}} f_{\sigma, c}$.

Next, we present reformulations for the constraints that are linear in the uncertainty $f$.

Proposition 3.4. Constraints (49) are satisfied if and only if the following constraints are satisfied:

$$
D_{C G}^{1} U_{s, C G}^{0}\left(1-\sum_{\sigma \in \mathcal{K}} \underline{f}_{\sigma, c^{1}} \delta_{\sigma}^{1}\right) \leq C A P_{C G} m_{s, C G}^{1}, \quad \forall s \in \mathcal{S} .
$$


Proof. We see that constraints (49) are equivalent to

$$
\begin{aligned}
& \max _{f^{1} \in \mathcal{F}} D_{C G}^{1} U_{s, C G}^{0}\left(1-\sum_{\sigma \in \mathcal{K}} f_{\sigma^{1}, c^{1}}^{1} \delta_{\sigma}^{1}\right) \leq C A P_{C G} m_{s, C G}^{1} & \forall s \in \mathcal{S}, \\
\Leftrightarrow & D_{C G}^{1} U_{s, C G}^{0}\left(1-\min _{f^{1} \in \mathcal{F}} \sum_{\sigma \in \mathcal{K}} f_{\sigma, c^{1}}^{1} \delta_{\sigma}^{1}\right) \leq C A P_{C G} m_{s, C G}^{1} & \forall s \in \mathcal{S} .
\end{aligned}
$$

From Proposition 3.2 and the definition of $\underline{f}$, we have

$$
\sum_{\sigma \in \mathcal{K}} \underline{f}_{\sigma, c^{1}} \tilde{\delta}_{\sigma}^{1}=\underline{f}_{\tilde{\sigma}^{1}, c^{1}}=\min _{f^{1} \in \mathcal{F}} f_{\tilde{\sigma}^{1}, c^{1}}^{1}=\min _{f^{1} \in \mathcal{F}} \sum_{\sigma \in \mathcal{K}} f_{\sigma, c^{1}}^{1} \tilde{\delta}_{\sigma}^{1} .
$$

which means constraints (49) are equivalent to constraints (54).

Proposition 3.5. Constraints (50) are satisfied if and only if the following constraints are satisfied:

$$
D_{N G}^{1}\left(U_{s, N G}^{0}+U_{s, C G}^{0} \sum_{\sigma \in \mathcal{K}} \bar{f}_{\sigma, c^{1}} \delta_{\sigma}^{1}\right) \leq C A P_{C G} m_{s, C G}^{1} \quad \forall s \in \mathcal{S}
$$

Proof. The proof relies on the definition of $\bar{f}$ and on arguments similar to those used in the proof of Proposition 3.4.

Next, we focus on the constraints of $\mathcal{M}^{\text {stat }}$ that involve quadratic dependencies on $f$.

Proposition 3.6. Constraints (51) are satisfied if and only if the following constraints are satisfied:

$$
\frac{D_{C G}^{2} U_{s, C G}^{0}}{C A P_{C G}}\left[1-\left(\sum_{\sigma^{1} \in \mathcal{K}} \underline{f}_{\sigma^{1}, c^{1}} \delta_{\sigma^{1}}^{1}+\sum_{\sigma^{2} \in \mathcal{K}} \sum_{c^{2} \in \mathcal{C}} \underline{f}_{\sigma^{2}, c^{2}} \tilde{\delta}_{\sigma^{2}}^{2} \gamma_{c^{2}}^{2}\left(1-\sum_{\sigma^{1} \in \mathcal{K}} \underline{f}_{\sigma^{1}, c^{1}} \delta_{\sigma^{1}}^{1}\right)\right)\right] \leq m_{s, C G}^{2} \quad \forall s \in \mathcal{S} .
$$

Proof. First, we see that if constraints (51) are satisfied, then constraints (56) are satisfied since $\underline{f} \in \mathcal{F}$.

Reciprocally, let us assume that constraints (56) are satisfied. Due to Propositions 3.2 and 3.3, we know that:

$$
\sum_{\sigma^{1} \in \mathcal{K}} f_{\sigma^{1}, c^{1}}^{1} \tilde{\delta}_{\sigma}^{1}+\sum_{\sigma^{2} \in \mathcal{K}} \sum_{c^{2} \in \mathcal{C}} f_{\sigma^{2}, c^{2}}^{2} \tilde{\delta}_{\sigma^{2}}^{2} \tilde{\gamma}_{c^{2}}^{2}\left(1-\sum_{\sigma^{1} \in \mathcal{K}} f_{\sigma^{1}, c^{1}}^{1} \tilde{\delta}_{\sigma}^{1}\right)=f_{\tilde{\sigma}^{1}, c^{1}}^{1}+f_{\tilde{\sigma}^{2}, \tilde{c}^{2}}^{2}\left(1-f_{\tilde{\sigma}^{1}, c^{1}}^{1}\right) .
$$

To simplify notations, let us denote $f_{\tilde{\sigma}^{1}, c^{1}}^{1}$ and $f_{\tilde{\sigma}^{2}, \tilde{c}^{2}}^{2}$ by $x$ and $y$ respectively. We also denote $\underline{f}_{\tilde{\sigma}^{1}, c^{1}}$ by $\underline{x}, \bar{f}_{\tilde{\sigma}^{1}, c^{1}}$ by $\bar{x}, \underline{f}_{\tilde{\sigma}^{2}, \tilde{c}^{2}}$ by $\underline{y}$ and $\bar{f}_{\tilde{\sigma}^{2}, \tilde{c}^{2}}$ by $\bar{y}$.

Recalling that $(x, y) \in[0,1]^{2}$, we wish to find out where function $g(x, y)=x+y(1-x)=x+y-x y$ defined on $(x, y) \in[\underline{x}, \bar{x}] \times[\underline{y}, \bar{y}] \subseteq[0,1]^{2}$ reaches its minimal value. First notice that

$$
g(x, y)=x+(1-x) y \geq x+(1-x) \underline{y}=g(x, \underline{y}) \quad \forall x \in[\underline{x}, \bar{x}], \forall y \in[\underline{y}, \bar{y}]
$$


and symmetrically $g(x, y) \geq g(\underline{x}, y), \forall x \in[\underline{x}, \bar{x}], \forall y \in[\underline{y}, \bar{y}]$. Consequently, $g(x, y) \geq g(\underline{x}, \underline{y}), \forall x \in$ $[\underline{x}, \bar{x}], \forall y \in[\underline{y}, \bar{y}]$, and we thus have:

$$
\begin{aligned}
& \max _{f^{1} \in \mathcal{F}, f^{2} \in \mathcal{F}} \frac{D_{C G}^{2} U_{s, C G}^{0}}{C A P_{C G}}\left[1-\left(\sum_{\sigma^{1} \in \mathcal{K}} f_{\sigma^{1}, c^{1}}^{1} \delta_{\sigma^{1}}^{1}+\sum_{\sigma^{2} \in \mathcal{K}} \sum_{c^{2} \in \mathcal{C}} f_{\sigma^{2}, c^{2}}^{2} \delta_{\sigma^{2}}^{2} \gamma_{c^{2}}^{2}\left(1-\sum_{\sigma^{1} \in \mathcal{K}} f_{\sigma^{1}, c^{1}}^{1} \delta_{\sigma^{1}}^{1}\right)\right)\right] \\
= & \frac{D_{C G}^{2} U_{s, C G}^{0}}{C A P_{C G}}\left[1-\min _{f^{1} \in \mathcal{F}, f^{2} \in \mathcal{F}}\left(\sum_{\sigma^{1} \in \mathcal{K}} f_{\sigma^{1}, c^{1}}^{1} \delta_{\sigma}^{1}+\sum_{\sigma^{2} \in \mathcal{K}} \sum_{c^{2} \in \mathcal{C}} f_{\sigma^{2}, c^{2}}^{2} \delta_{\sigma^{2}}^{2} \gamma_{c^{2}}^{2}\left(1-\sum_{\sigma^{1} \in \mathcal{K}} f_{\sigma^{1}, c^{1}}^{1} \delta_{\sigma}^{1}\right)\right)\right] \\
\leq & \frac{D_{C G}^{2} U_{s, C G}^{0}}{C A P_{C G}}\left[1-\left(\sum_{\sigma^{1} \in \mathcal{K}} \underline{f}_{\sigma^{1}, c^{1}} \delta_{\sigma}^{1}+\sum_{\sigma^{2} \in \mathcal{K}} \sum_{c^{2} \in \mathcal{C}} \underline{f}_{\sigma^{2}, c^{2}} \delta_{\sigma^{2}}^{2} \gamma_{c^{2}}^{2}\left(1-\sum_{\sigma^{1} \in \mathcal{K}} \underline{f}_{\sigma^{1}, c^{1}} \delta_{\sigma}^{1}\right)\right)\right] \leq m_{s, C G}^{2},
\end{aligned}
$$

which means that constraints (51) are satisfied.

Proposition 3.7. Constraints (53) are satisfied if and only if the following constraints are satisfied:

$$
\sum_{s \in \mathcal{S}}\left[U_{s, N G}^{0}+U_{s, C G}^{0}\left(\sum_{\sigma^{1} \in \mathcal{K}} \underline{f}_{\sigma^{1}, c^{1}} \delta_{\sigma^{1}}^{1}+\sum_{\sigma^{2} \in \mathcal{K}} \sum_{c^{2} \in \mathcal{C}} \underline{f}_{\sigma^{2}, c^{2}} \delta_{\sigma^{2}}^{2} \gamma_{c^{2}}^{2}\left(1-\sum_{\sigma^{1} \in \mathcal{K}} \underline{f}_{\sigma^{1}, c^{1}} \delta_{\sigma^{1}}^{1}\right)\right)\right] \geq \underline{Q o E} U^{t o t} .
$$

Proof. The proof relies on arguments similar to those used in the proof of Proposition 3.6.

Proposition 3.8. Constraints (52) are satisfied if and only if the following constraints are satisfied:

$$
\begin{gathered}
\frac{D_{N G}^{2}}{C A P_{N G}}\left[U_{s, N G}^{0}+U_{s, C G}^{0}\left(\sum_{\sigma^{1} \in \mathcal{K}} \bar{f}_{\sigma^{1}, c^{1}} \delta_{\sigma^{1}}^{1}+\sum_{\sigma^{2} \in \mathcal{K}} \sum_{c^{2} \in \mathcal{C}} \bar{f}_{\sigma^{2}, c^{2}} \delta_{\sigma^{2}}^{2} \gamma_{c^{2}}^{2}\left(1-\sum_{\sigma^{1} \in \mathcal{K}} \bar{f}_{\sigma^{1}, c^{1}} \delta_{\sigma^{1}}^{1}\right)\right)\right] \leq m_{s, N G}^{2} \\
\forall s \in \mathcal{S}
\end{gathered}
$$

Proof. The proof relies on studying the maximum of function $g$ and on arguments similar to those used in the proof of Proposition 3.6.

We consider next constraints (48) which require an argument slightly more involved.

Proposition 3.9. Constraints (48) are satisfied if and only if the following constraints are satisfied:

$$
\begin{aligned}
& \sum_{\sigma^{1} \in \mathcal{K}} \sigma^{1} \bar{f}_{\sigma^{1}, c^{1}} \delta_{\sigma^{1}}^{1}+\sum_{\sigma^{2} \in \mathcal{K}} \sum_{c^{2} \in \mathcal{C}} \sigma^{2} \bar{f}_{\sigma^{2}, c^{2}} \delta_{\sigma^{2}}^{2} \gamma_{c^{2}}^{2}\left(1-\sum_{\sigma^{1} \in \mathcal{K}} \bar{f}_{\sigma^{1}, c^{1}} \delta_{\sigma}^{1}\right) \leq \frac{C_{\text {sub }}}{\sum_{s \in \mathcal{S}} U_{s, C G}^{0}} \\
& \sum_{\sigma^{1} \in \mathcal{K}} \sigma^{1} \underline{f}_{\sigma^{1}, c^{1}} \delta_{\sigma^{1}}^{1}+\sum_{\sigma^{2} \in \mathcal{K}} \sum_{c^{2} \in \mathcal{C}} \sigma^{2} \bar{f}_{\sigma^{2}, c^{2}} \delta_{\sigma^{2}}^{2} \gamma_{c^{2}}^{2}\left(1-\sum_{\sigma^{1} \in \mathcal{K}} \underline{f}_{\sigma^{1}, c^{1}} \delta_{\sigma^{1}}^{1}\right) \leq \frac{C_{s u b}}{\sum_{s \in \mathcal{S}} U_{s, C G}^{0}}
\end{aligned}
$$


Proof. First, we see that if constraints (48) are satisfied, constraints (59) and (60) are satisfied since $\underline{f} \in \mathcal{F}$ and $\bar{f} \in \mathcal{F}$.

Reciprocally, let us assume that constraints (59) and (60) are both satisfied: due to Propositions 3.2 and 3.3 , we know that

$$
\sum_{\sigma^{1} \in \mathcal{K}} \sigma^{1} f_{\sigma^{1}, c^{1}}^{1} \tilde{\delta}_{\sigma^{1}}^{1}+\sum_{\sigma^{2} \in \mathcal{K}} \sum_{c^{2} \in \mathcal{C}} \sigma^{2} f_{\sigma^{2}, c^{2}}^{2} \tilde{\delta}_{\sigma^{2}}^{2} \tilde{\gamma}_{c^{2}}^{2}\left(1-\sum_{\sigma^{1} \in \mathcal{K}} f_{\sigma^{1}, c^{1}}^{1} \tilde{\delta}_{\sigma^{1}}^{1}\right)=\tilde{\sigma}^{1} f_{\tilde{\sigma}^{1}, c^{1}}^{1}+\tilde{\sigma}^{2} f_{\tilde{\sigma}^{2}, \tilde{c}^{2}}^{2}\left(1-f_{\tilde{\sigma}^{1}, c^{1}}^{1}\right) .
$$

We use the same notations as those introduced in the proof of Proposition 3.6. In addition, we denote $\tilde{\sigma}^{1}$ by $a$ and $\tilde{\sigma}^{2}$ by $b$. We wish to find out where function $h(x, y)=a x+b y(1-x)$ defined on $x \in[\underline{x}, \bar{x}] \times[\underline{y}, \bar{y}] \subseteq[0,1]^{2}$ reaches its maximal value with $a$ and $b$ positive reals. First notice that we have:

$$
h(x, y)=a x+b y(1-x) \leq a x+b \bar{y}(1-x)=h(x, \bar{y}) \quad \forall x \in[\underline{x}, \bar{x}], \forall y \in[\underline{y}, \bar{y}] .
$$

Hence, our function is maximal for $y=\bar{y}$. With $y$ fixed to $\bar{y}, h(x, \bar{y})$ becomes a linear function of $x$ defined on $[\underline{x}, \bar{x}]$. Therefore,

$$
h(x, \bar{y}) \leq \max \{h(\bar{x}, \bar{y}), h(\underline{x}, \bar{y})\} \quad \forall x \in[\underline{x}, \bar{x}]
$$

and

$$
h(x, y) \leq \max \{h(\bar{x}, \bar{y}), h(\underline{x}, \bar{y})\} \quad \forall x \in[\underline{x}, \bar{x}], \forall y \in[\underline{y}, \bar{y}] .
$$

We thus have for each $f^{1} \in \mathcal{F}, f^{2} \in \mathcal{F}$ :

$$
\begin{aligned}
& \sum_{\sigma^{1} \in \mathcal{K}} \sigma^{1} f_{\sigma^{1}, c^{1}}^{1} \delta_{\sigma^{1}}^{1}+\sum_{\sigma^{2} \in \mathcal{K}} \sum_{c^{2} \in \mathcal{C}} \sigma^{2} f_{\sigma^{2}, c^{2}}^{2} \delta_{\sigma^{2}}^{2} \gamma_{c^{2}}^{2}\left(1-\sum_{\sigma^{1} \in \mathcal{K}} f_{\sigma^{1}, c^{1}}^{1} \delta_{\sigma^{1}}^{1}\right) \\
& \leq \max \left\{\sum_{\sigma^{1} \in \mathcal{K}} \sum_{c^{1} \in \mathcal{C}} \sigma^{1} \bar{f}_{\sigma^{1}, c^{1}} \delta_{\sigma^{1}}^{1}+\sum_{\sigma^{2} \in \mathcal{K}} \sum_{c^{2} \in \mathcal{C}} \sigma^{2} \bar{f}_{\sigma^{2}, c^{2}} \delta_{\sigma^{2}}^{2} \gamma_{c^{2}}^{2}\left(1-\sum_{\sigma^{1} \in \mathcal{K}} \sum_{c^{1} \in \mathcal{C}} \bar{f}_{\sigma^{1}, c^{1}} \delta_{\sigma^{1}}^{1}\right),\right. \\
&\left.\sum_{\sigma^{1} \in \mathcal{K}} \sum_{c^{1} \in \mathcal{C}} \sigma^{1} \underline{f}_{\sigma^{1}, c^{1}} \delta_{\sigma}^{1}+\sum_{\sigma^{2} \in \mathcal{K}} \sum_{c^{2} \in \mathcal{C}} \sigma^{2} \bar{f}_{\sigma^{2}, c^{2}} \delta_{\sigma^{2}}^{2} \gamma_{c^{2}}^{2}\left(1-\sum_{\sigma^{1} \in \mathcal{K}} \sum_{c^{1} \in \mathcal{C}} \underline{f}_{\sigma^{1}, c^{1}} \delta_{\sigma^{1}}^{1}\right)\right\} \leq \frac{C_{s u b}}{\sum_{s \in \mathcal{S}} U_{s, C G}^{0}},
\end{aligned}
$$

which means that constraints (48) are satisfied.

Summarizing the above results, we have shown that formulation $\mathcal{M}^{\text {stat }}$ is equivalent to the following mixed-integer linear programming, with a finite number of constraints:

$$
\min \quad\{(47) \quad \text { s.t. } \quad(2)-(4),(31),(34),(54)-(60),(13)-(26)\}
$$




\subsection{Adjustable robust counterpart}

In this section, we consider the framework where the operator can take benefit from the knowledge of the uncertainty realization in the first period when deciding the subsidy offered in the second period. We obtain a two-stage model where the operator can adapt to the uncertainty through a second-stage decision: the amount of subsidy offered in the second period. We model this decision by variables $\delta_{\sigma}^{2}\left(f^{1}\right)$ defined for each $f^{1} \in \mathcal{F}$. Let us recall that all decisions concerning network investments are taken as planning decisions and considered to be taken in the first-stage, which means that $\delta_{\sigma}^{2}\left(f^{1}\right)$ is the only second-stage decision. This decision is often called the recourse in the robust optimization literature.

Plugging the recourse variables into the robust model leads to a mixed-integer formulation with an infinite number of variables since one variable $\delta\left(f^{\prime}\right)$ arises for each $f^{\prime} \in \mathcal{F}$. Therefore, we propose an adjustable robust partition method in the line of Bertsimas et al. (2015), splitting our uncertainty set $\mathcal{F}$ into a finite number $L$ of parts. Notice that, unlike Bertsimas et al. (2015), we partition the uncertainty set from the start. We denote the index set of the partition by $\mathcal{L}=\{1, \ldots, L\}$. We hence have $\mathcal{F}=\cup_{l=0}^{L} \mathcal{F}(l)$ with $\forall l \in \mathcal{L}, l^{\prime} \in \mathcal{L} \backslash\{l\}, \mathcal{F}(l) \cap \mathcal{F}\left(l^{\prime}\right)=\emptyset$ and consider the piecewise constant recourse defined by:

$$
\delta^{2}(f)=\delta^{2}\left(f^{\prime}\right) \quad \forall f, f^{\prime} \in \mathcal{F}(l), \forall l \in \mathcal{L}
$$

Thus, we replace $\delta_{\sigma}^{2}(f)$ by $\delta_{\sigma, l}^{2}$ which indicates the recourse decision taken when $f \in \mathcal{F}(l)$ for each $l \in \mathcal{L}$. Consequently, the robust adjustable counterpart of formulation $\mathcal{M}^{\text {det,2p }}$ can be written as follows:

$$
\begin{aligned}
& \min C_{\text {sub }}+\sum_{s \in \mathcal{S}} \sum_{g \in \mathcal{G}} C M_{g}\left(m_{s, g}^{2}-M_{s, g}^{0}\right)+\sum_{s \in \mathcal{S}} C A_{N G}\left(z_{s, N G}^{2}-Z_{s, N G}^{0}\right) \\
& \text { s.t. } \quad(2)-(4),(31),(34),(49)-(50),(14)-(25) \\
& \sum_{\sigma^{1} \in \mathcal{K}} \sigma^{1} f_{\sigma^{1}, c^{1}}^{1} \delta_{\sigma}^{1}+\sum_{\sigma^{2} \in \mathcal{K}} \sum_{c^{2} \in \mathcal{C}} \sigma^{2} f_{\sigma^{2}, c^{2}}^{2} \delta_{\sigma^{2}, l}^{2} \gamma_{c^{2}}^{2}\left(1-\sum_{\sigma^{1} \in \mathcal{K}} f_{\sigma^{1}, c^{1}}^{1} \delta_{\sigma}^{1}\right) \leq \frac{C_{s u b}}{\sum_{s \in \mathcal{S}} U_{s, C G}^{0}} \quad \forall l \in \mathcal{L}, \forall f^{1} \in \mathcal{F}(l), \forall f^{2} \in \mathcal{F}, \\
& \\
& \frac{D_{C G}^{2} U_{s, C G}^{0}}{C A P_{C G}}\left[1-\left(\sum_{\sigma^{1} \in \mathcal{K}} f_{\sigma^{1}, c^{1}}^{1} \delta_{\sigma}^{1}+\sum_{\sigma^{2} \in \mathcal{K}} \sum_{c^{2} \in \mathcal{C}} f_{\sigma^{2}, c^{2}}^{2} \delta_{\sigma^{2}, l}^{2} \gamma_{c^{2}}^{2}\left(1-\sum_{\sigma^{1} \in \mathcal{K}} f_{\sigma^{1}, c^{1}}^{1} \delta_{\sigma}^{1}\right)\right)\right] \leq m_{s, C G}^{2} \\
& \frac{D_{N G}^{2}}{C A P_{N G}}\left[U_{s, N G}^{0}+U_{s, C G}^{0}\left(\sum_{\sigma^{1} \in \mathcal{K}} f_{\sigma^{1}, c^{1}}^{1} \delta_{\sigma}^{1}+\sum_{\sigma^{2} \in \mathcal{K}} \sum_{c^{2} \in \mathcal{C}} f_{\sigma^{2}, c^{2}}^{2} \delta_{\sigma^{2}, l}^{2} \gamma_{c^{2}}^{2}\left(1-\sum_{\sigma^{1} \in \mathcal{K}} f_{\sigma^{1}, c^{1}}^{1} \delta_{\sigma}^{1}\right)\right)\right] z_{s, N G}^{2} \leq m_{s, N G}^{2}
\end{aligned}
$$




$$
\begin{aligned}
& \forall s \in \mathcal{S}, \forall l \in \mathcal{L}, \forall f^{1} \in \mathcal{F}(l), \forall f^{2} \in \mathcal{F} \\
& \sum_{s \in \mathcal{S}}\left[U_{s, N G}^{0}+U_{s, C G}^{0}\left(\sum_{\sigma^{1} \in \mathcal{K}} f_{\sigma^{1}, c^{1}}^{1} \delta_{\sigma}^{1}+\sum_{\sigma^{2} \in \mathcal{K}} \sum_{c^{2} \in \mathcal{C}} f_{\sigma^{2}, c^{2}}^{2} \delta_{\sigma^{2}, l}^{2} \gamma_{c^{2}}^{2}\left(1-\sum_{\sigma^{1} \in \mathcal{K}} f_{\sigma^{1}, c^{1}}^{1} \delta_{\sigma^{1}}^{1}\right)\right)\right] z_{s, N G}^{2} \geq \underline{Q o E} U^{\text {tot }}, \\
& \forall l \in \mathcal{L}, \forall f^{1} \in \mathcal{F}(l), \forall f^{2} \in \mathcal{F}, \\
& \sum_{\sigma \in \mathcal{K}} \delta_{\sigma}^{1}=1, \\
& \sum_{\sigma \in \mathcal{K}} \delta_{\sigma, l}^{2}=1 \\
& \delta_{\sigma}^{1} \in\{0,1\} \\
& \forall \sigma \in \mathcal{K}, \\
& \delta_{\sigma, l}^{2} \in\{0,1\} \\
& \forall \sigma \in \mathcal{K}, \forall l \in \mathcal{L} \text {. }
\end{aligned}
$$

Let us denote this formulation by $\mathcal{M}^{a d j, L}$ where $L$ is the number of parts. Formulation $\mathcal{M}^{a d j, L}$ is obtained by replacing each set of constraints involving the second period in formulation $\mathcal{M}^{\text {stat }}$ (constraints (48),(51)-(53),(13)) with $L$ sets of constraints - one for each part. We observe that in each part $\mathcal{F}(l), l \in \mathcal{L}$, constraints (62)-(65) can be reformulated in the same way as for the static model, with in the first period $\underline{f}$ and $\bar{f}$ replaced by $\underline{f}(l)$ and $\bar{f}(l)$ defined by $\underline{f}_{\sigma, c}(l)=\min _{f \in \mathcal{F}(l)} f_{\sigma, c}$ and $\bar{f}_{\sigma, c}(l)=\max _{f \in \mathcal{F}(l)} f_{\sigma, c}$, for each $l \in \mathcal{L}$. We notice that formulation $\mathcal{M}^{a d j, 1}$ is equivalent to formulation $\mathcal{M}^{\text {stat }}$.

Remark 3.10. As for the deterministic and static models, formulations $\mathcal{M}^{a d j, L}$ can be linearized and reinforced by RLT equalities. The only slight difference is that for each linearization or equality implying variables $\delta_{\sigma}^{2}$ in the deterministic model, we have now $L$ constraints corresponding to each part.

So far we have proposed to use a static partition of the uncertainty polytope. Such an approach could, for instance, split each coordinate $f_{\sigma, c} \in\left[\underline{f}_{\sigma, c}, \bar{f}_{\sigma, c}\right]$ into $\widehat{L}$ pieces of equal length, for some positive integer $\widehat{L}$. Let us investigate this idea further and denote by $I_{\sigma, c}\left(\lambda_{\sigma, c}\right)$ the $\lambda_{\sigma, c^{-}}$th subinterval of $\left[\underline{f}_{\sigma, c}, \bar{f}_{\sigma, c}\right]$ resulting from the split for each $\sigma \in \mathcal{K}$ and $c \in \mathcal{C}$. Then, concatenating all values $\lambda_{\sigma, c}$ into the tuple $\lambda$, we define

$$
\mathcal{F}(\lambda)=\mathcal{F} \bigcap_{\substack{\sigma \in \mathcal{K} \\ c \in \mathcal{C}}} I_{\sigma, c}\left(\lambda_{\sigma, c}\right),
$$

and obtain the partition

$$
\left\{\mathcal{F}(\lambda): \lambda_{\sigma, c}=1, \ldots, \widehat{L}, \sigma \in \mathcal{K}, c \in \mathcal{C}\right\}
$$




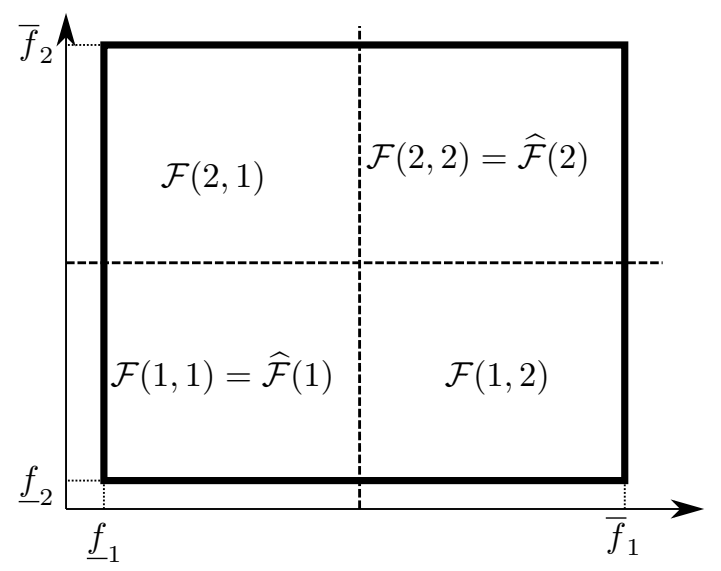

Figure 1: Example of splitting when $\mathcal{F}$ is a 2-dimensional box, $\widehat{L}=2$, and $L=4$.

which may contain empty elements whenever $\mathcal{F}$ is not a box. Partition $(70)$ contains $L=\widehat{L}^{|\mathcal{K}| \cdot|\mathcal{C}|}$ elements, so it scales exponentially with the dimension of the uncertainty polytope $\mathcal{F}$ and is unlikely to be of any practical use. In what follows, we overcome this difficulty by assuming that $\mathcal{F}$ is a box and considering only a relevant subset of $\mathcal{F}(\lambda)$.

Proposition 3.11. Let us define $\widehat{\mathcal{L}}=\{1, \ldots, \widehat{L}\}$ and introduce $\widehat{\mathcal{F}}(\hat{\lambda})=\mathcal{F}(\hat{\lambda}, \ldots, \hat{\lambda})$ for $\hat{\lambda} \in \widehat{\mathcal{L}}$. Let $\widehat{\mathcal{M}}^{\text {adj, }} \widehat{L}$ be the counterpart of formulation $\mathcal{M}^{\text {adj,L }}$ applied to uncertainty set $\widehat{\mathcal{F}}=\bigcup_{\hat{\lambda} \in \widehat{\mathcal{L}}} \widehat{\mathcal{F}}(\hat{\lambda})$ together with the partition induced by $\widehat{\mathcal{L}}$. If $\mathcal{F}$ is a box, any first-stage solution is feasible for problem $\mathcal{M}^{\text {adj, } L}$ with partition (70) if and only if it is feasible for problem $\widehat{\mathcal{M}}^{\text {adj }}, \widehat{L}$.

Proof. Notice that $\widehat{\mathcal{F}}(\hat{\lambda})=\mathcal{F}(\lambda)$ for $\lambda_{\sigma, c}=\hat{\lambda}$ for each $\sigma \in \mathcal{K}$ and $c \in \mathcal{C}$, so that formulation $\mathcal{M}^{\text {adj,L }}$ involves all constraints of formulation $\widehat{\mathcal{M}}^{\text {adj }, \widehat{L}}$. Therefore, any first-stage solution feasible for $\mathcal{M}^{\text {adj,L }}$ is feasible for $\widehat{\mathcal{M}}^{\text {adj, }} \widehat{L}$.

To prove the reverse relation, consider any solution $\hat{z}, \hat{m}, \hat{\alpha}, \hat{\delta}, \hat{\sigma}, \hat{\gamma}$ feasible for $\widehat{\mathcal{M}}^{\text {adj, }}, \widehat{L}$ and let $f_{\hat{\sigma}^{1}, c^{1}}^{1}$ be the non-zero term from the sum $\sum_{\sigma \in \mathcal{K}} f_{\sigma, c^{1}}^{1} \hat{\delta}_{\sigma}^{1}$ (see Proposition 3.2). To define a second-stage solution $\delta^{2}$ feasible for $\mathcal{M}^{a d j, L}$, consider $\lambda \in \mathcal{L}$. Following the above discussion, only the projection of $\mathcal{F}(\lambda)$ on the component $\left(\hat{\sigma}^{1}, c^{1}\right)$ impacts the constraints of $\mathcal{M}^{\text {adj,L}}$. Therefore, we take $\hat{\lambda}=\lambda_{\hat{\sigma}^{1}, c^{1}}$ and define $\delta_{\sigma, \lambda}^{2}=\delta_{\sigma, \hat{\lambda}}^{2}$. Proceeding that way for all $\lambda \in \mathcal{L}$, we obtain a second-stage solution $\delta^{2}$ which, together with first-stage solution $\hat{z}, \hat{m}, \hat{\alpha}, \hat{\delta}^{1}, \hat{\sigma}, \hat{\gamma}$, is feasible for $\mathcal{M}^{\text {adj,L}}$.

Figure 1 illustrates the relationship between $\mathcal{F}(\lambda)$ and $\widehat{\mathcal{F}}(\hat{\lambda})$ in a two-dimensional context. 
Furthermore, we provide an example of construction $\widehat{\mathcal{F}}(\hat{\lambda})$ in the next section, after defining the specific uncertainty set $\mathcal{F}$ used in our experiments. Notice that while $\widehat{\mathcal{F}}$ is not a convex set, this does not impact our solution approach since each set $\widehat{\mathcal{F}}(\hat{\lambda})$ is considered independently in $\widehat{\mathcal{M}}^{\text {adj, }} \widehat{L}$.

\section{Case-study and uncertainty definition}

In this section, we illustrate the potential benefit of the robust static and adjustable models. First, we compare their optimal costs to the one of the deterministic solution. Second, we run simulations for assessing the quality of the obtained first period decisions in a realistic context. We first describe in Section 4.1 the uncertainty set used in the experiments. The instances used and computational settings are described in Section 4.2. Section 4.3 presents the results and the process used for the simulations.

\subsection{Design of the uncertainty set}

Let $\tilde{f}_{\sigma, c}$ denote the nominal value of $f_{\sigma, c}$. We define an uncertainty set that satisfies two requirements: (i) it allows for a controlled variation around the nominal value, and (ii) its size is the same for all possibles values of $\tilde{f}_{\sigma, c} \in[0,1]$. Thus, letting $\Gamma$ denote the amplitude of the variation, we define

$$
\mathcal{F}=\left\{f \in[0,1]^{|C| \cdot|K|} \mid(1-\Gamma) \tilde{f}_{\sigma, c} \leq f_{\sigma, c} \leq(1-\Gamma) \tilde{f}_{\sigma, c}+\Gamma, c \in \mathcal{C}, \sigma \in \mathcal{K}\right\}
$$

An illustration of $\mathcal{F}$ for a nominal function corresponding to the reference example from Cambier et al. (2020) and $\Gamma=0.25$ is provided in Figure 2. We see that the uncertainty set is not symmetric around the nominal value $\tilde{f}_{\sigma, c}$ as the downward deviation from $\tilde{f}_{\sigma, c}$ can be as large as $\Gamma \tilde{f}_{\sigma, c}$ while the upward deviation is bounded by $\Gamma\left(1-\tilde{f}_{\sigma, c}\right)$ for $\sigma \in \mathcal{K}$ and $c \in \mathcal{C}$, with these deviations depending on the nominal value. This definition of the uncertainty set enables us to control that each possible reaction belongs to $[0,1]$.

Following the discussions of Section 3.3 , we define for each $\hat{\lambda}$ in $\{1, \ldots, \widehat{L}\}$

$$
\widehat{\mathcal{F}}(\hat{\lambda})=\left\{f \in[0,1]^{|C| \cdot|K|} \mid(1-\Gamma) \tilde{f}_{\sigma, c}+\frac{\hat{\lambda}-1}{L} \Gamma \leq f_{\sigma, c} \leq(1-\Gamma) \tilde{f}_{\sigma, c}+\frac{\hat{\lambda}}{L} \Gamma, c \in \mathcal{C}, \sigma \in \mathcal{K}\right\} \subseteq \mathcal{F} .
$$

An illustration of the splitting for $\widehat{L}=3$ and the example from Figure 2 is provided in Figure 3 . 

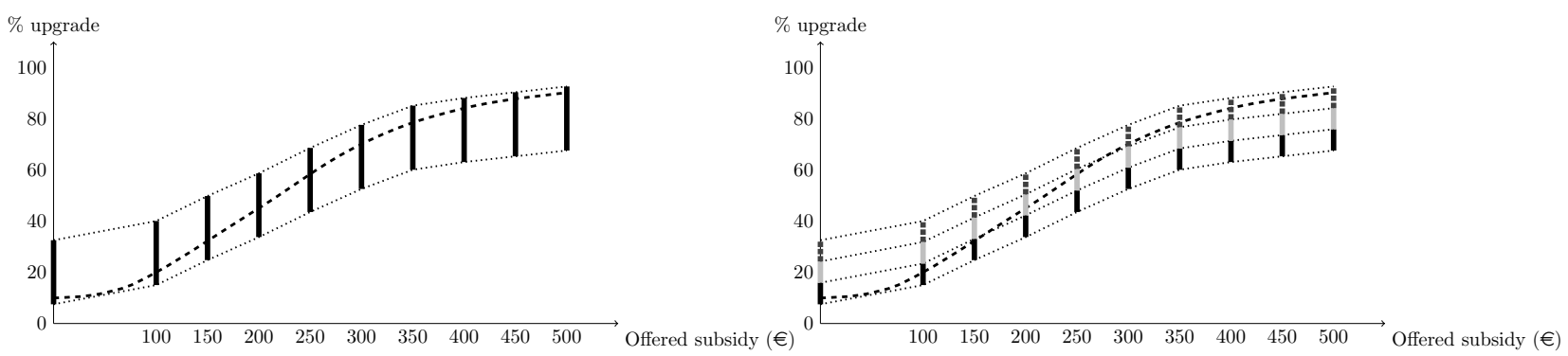

Figure 2: Example of uncertainty set with reference

Figure 3: Example of split $(\widehat{L}=3)$ uncertainty set with function from Cambier et al. (2020) and $\Gamma=0.25$ (projected on a given range $c=[0.75,1]$ ). reference function from Cambier et al. (2020) and $\Gamma=$ 0.25 (projected on a given range $c=[0.75,1]$ )

\subsection{Instances parameters}

We aim to assess the different models presented in Section 2 on a realistic instance for three different amplitude values $\Gamma$. We use the $3 \mathrm{G} / 4 \mathrm{G}$ suburban instance of 100 sites from Cambier et al. (2020), adapted for a two period framework. At the beginning of the time-horizon, 34 sites are equipped with both $3 \mathrm{G}$ and $4 \mathrm{G}$ technologies while 66 are 3G-only sites. The network parameter values are realistic values taken from telecommunication equipment sellers. The strategic guideline for quality of experience $\underline{Q o E}$ is fixed to 0.8 and a $N G$ coverage target of $\underline{\alpha}=0.7$, i.e. 70 sites, is asked. Regarding the subscriber dynamic, values for $\tilde{f}_{\sigma, c}$ are given in Table 3 . We use the

\begin{tabular}{|c|c|c|c|c|c|c|c|c|c|c|}
\hline Coverage level \Subsidies (in $€$ ) & 0 & 100 & 150 & 200 & 250 & 300 & 350 & 400 & 450 & 500 \\
\hline medium low (25-50\%) & 0.5 & 5 & 12 & 21 & 30 & 40 & 42 & 43 & 44 & 45 \\
\hline medium high (50-75\%) & 5 & 10 & 20 & 30 & 40 & 50 & 60 & 62 & 64 & 65 \\
\hline high (75-100\%) & 10 & 20 & 33 & 45 & 58 & 70 & 80 & 83 & 87 & 90 \\
\hline
\end{tabular}

Table 3: Reaction of the subscribers (in \%) on reference markets for given subsidies and coverage levels.

uncertainty set for $f$ defined by these nominal values and an amplitude $\Gamma \in\{0.25,0.30,0.35\}$.

The computations have been made on a server of 32 processors Intel Xeon of CPU 5110 clocked at $1.6 \mathrm{GHz}$ each. The code is written in Julia 1.1.0, with the package JuMP developed for discrete optimization (see Dunning et al. (2017) for details) and the solver used is CPLEX 12.8 (default branch-and-bound algorithm). The time limit for MILP solving is set to 7200 seconds. 
Table 4: Solutions for $0.25,0.3$ and 0.35 deviations

\begin{tabular}{|c|c|c|c|c|c|c|c|c|c|c|c|c|}
\hline$\Gamma$ & model & sol & time & first-stage $\left(\sigma^{1}\right)$ & \multicolumn{8}{|c|}{ second-stage $\left(\sigma^{2}\right)$} \\
\hline & deterministic & 6999 & 19 & 200 & \multicolumn{8}{|c|}{250} \\
\hline \multirow[t]{4}{*}{0.25} & static & 9691 & 18 & 250 & \multicolumn{8}{|c|}{350} \\
\hline & $\operatorname{adj}(\widehat{L}=2)$ & 9643 & 78 & 350 & \multicolumn{4}{|c|}{300} & \multicolumn{4}{|c|}{250} \\
\hline & $\operatorname{adj}(\widehat{L}=4)$ & 9643 & 347 & 350 & \multicolumn{2}{|c|}{300} & \multicolumn{2}{|c|}{250} & \multicolumn{2}{|c|}{250} & \multicolumn{2}{|c|}{200} \\
\hline & $\operatorname{adj}(\widehat{L}=8)$ & 9580 & 7200 & 350 & 300 & 300 & 300 & 250 & 250 & 250 & 200 & 200 \\
\hline \multirow[t]{4}{*}{0.30} & static & 10097 & 10 & 300 & \multicolumn{8}{|c|}{350} \\
\hline & $\operatorname{adj}(\widehat{L}=2)$ & 10066 & 36 & 350 & \multicolumn{4}{|c|}{300} & \multicolumn{4}{|c|}{250} \\
\hline & $\operatorname{adj}(\widehat{L}=4)$ & 10066 & 274 & 350 & \multicolumn{2}{|c|}{300} & \multicolumn{2}{|c|}{300} & \multicolumn{2}{|c|}{250} & \multicolumn{2}{|c|}{150} \\
\hline & $\operatorname{adj}(\widehat{L}=8)$ & 9965 & 3095 & 350 & 300 & 300 & 300 & 250 & 250 & 250 & 150 & 250 \\
\hline \multirow[t]{4}{*}{0.35} & static & 10957 & 8 & 350 & \multicolumn{8}{|c|}{350} \\
\hline & $\operatorname{adj}(\widehat{L}=2)$ & 10767 & 28 & 350 & \multicolumn{4}{|c|}{350} & \multicolumn{4}{|c|}{250} \\
\hline & $\operatorname{adj}(\widehat{L}=4)$ & 10671 & 130 & 350 & \multicolumn{2}{|c|}{350} & \multicolumn{2}{|c|}{300} & \multicolumn{2}{|c|}{250} & \multicolumn{2}{|c|}{200} \\
\hline & $\operatorname{adj}(\widehat{L}=8)$ & 10671 & 1105 & 350 & 350 & 350 & 300 & 300 & 300 & 200 & 150 & 100 \\
\hline
\end{tabular}

\subsection{Results}

\subsubsection{Solutions of the formulations}

Results obtained with each formulation are presented in Table 4. The value of the best solution found and the solving time in seconds are provided respectively in columns "sol" and "time" of Table 4. Column "first-stage" stands for the value of the subsidy offered in the first period and multi-column "second-stage" for the value of the subsidy offered in the second period. For the adjustable models, each line presents second-stage values ordered from lowest to highest reaction case.

On a scalability viewpoint, we observe that the convergence of the branch-and-bound procedure is obtained in less than 2 minutes for the deterministic, static and adjustable $(\widehat{L}=2)$ models for each amplitude value. For each model, the larger the amplitude is, the faster the convergence is. We also observe that whatever the amplitude is, the static model is not harder to solve than the deterministic model. Refining the number of parts considerably increases the model size (in terms of constraints and variables). Our results enlighten the lack of scalability of such refinements. For $\Gamma=0.25$ and $\widehat{L}=8$, the solver does not converge within the two-hours time limit.

Next, we observe that the deterministic decision for the first-level (200€) is never taken by any of the robust frameworks. We see that for an amplitude $\Gamma=0.25$, the first-stage decision for the 
subsidy proposal is different between the static and adjustable models: static ( $250 €)$ and adjustable $(350 €$ for all values of $\widehat{L})$. For $\Gamma=0.30$ deviation, the first-stage decisions for the subsidy proposal are $300 €$ for the static model and $350 €$ for all adjustable models. These differences are explained by an impact on network installations (fewer installations) in the adjustable cases. For $\Gamma=0.35$ deviation, the decision for the static and all adjustable models is the same: $350 €$. From our results, we conclude that using the adjustable model with $\widehat{L}=2$ is sufficient since it converges a lot faster than the adjustable model with $\widehat{L}=4$ and $\widehat{L}=8$ and provides the same first-stage solution in each case.

\subsubsection{Simulation}

In practice, operators solve the models (deterministic, static, or adjustable) and implement only the first period decisions obtained. Then, upon knowledge of the true reactions to subsidies, they decide of the decisions in the second period. Having this in mind, we assess the solutions returned by the different models as follows. Consider the first period decisions of the solution returned by one of the three models, be it deterministic, static, or adjustable (using a 2-partition). Let $\tilde{\sigma}^{1}$ and $\tilde{c}^{1}$ be the subsidy and the range of coverage in the first period in that solution, the corresponding value of the reaction is $f_{\tilde{\sigma}^{1}, \tilde{c}^{1}}^{1}$. Note that this is an uncertain value. Next, we sample 200 different values for $f_{\tilde{\sigma}^{1}, \tilde{c}^{1}}^{1}$ spanning the whole uncertainty set $\left(f_{\tilde{\sigma}^{1}, \tilde{c}^{1}}^{1} \in \mathcal{F}_{S},\left|\mathcal{F}_{S}\right|=200\right)$ : each subsequent pair of samples is distant of $1 /(\Gamma-1)$ ). For each such $f_{\tilde{\sigma}^{1}, \tilde{c}^{1}}^{1} \in \mathcal{F}_{S}$, we compute the optimal second-stage decisions with a variant of formulation $\mathcal{M}^{\text {stat }}$, imposing the first period decisions and constraining the first period reaction to be equal to the sampled value.

The simulations costs (curve formed by all generated scenarios) are plotted for each value of $\Gamma$ on Figures 4, 5 and 6. The worst-case costs are given under column "worst-case cost" in Table 5. The curves show that these worst-case costs are obtained for the scenarios where the uncertainty is the lower, as these functions are mostly decreasing. We see that for the worst-case of each amplitude value, the first-stage decision of the adjustable model is the best one. Said differently, adjustable robust solutions are less risky than the other ones. More importantly, we observe that for $\Gamma=0.35$, the deterministic first-stage decision is infeasible, see Table 5 and the missing values for the deterministic curve in Figure 6.

On an economic viewpoint, we first notice from Table 4 that the optimal cost from the deterministic framework (6999 $\mathrm{k} €$ ) is around 30\% lower than the best solution found (obtained with 
the adjustable model) in the worst-case (for $\Gamma=0.25$ ). This can be explained by computing the worst-case for the $Q o E$ threshold when $\Gamma=0.25$ and the decisions taken by the deterministic model are imposed. This value is equal to 0.71 while the demanded threshold is 0.8 . Deterministic decisions lead hence to infeasibility in the robust context, which requires higher subsidies for robust decisions. The impact on the cost in a robust context is three-fold. It increases,

- since the subsidy proposal is higher,

- since these subsidies have a higher nominal effect (more reactions due to decisions),

- if the subscribers react better than expected (more reactions due to uncertainty).

Table 5: Simulated cost for $0.25,0.3$ and 0.35 deviations

\begin{tabular}{|c|c|c|c|}
\hline$\Gamma$ & model for first-stage & first-stage $\left(\sigma^{1}\right)$ & worst-case cost \\
\hline 0.25 & deterministic & 200 & 10671 \\
0.25 & static & 250 & 9659 \\
0.25 & adj & 350 & 9270 \\
\hline 0.30 & deterministic & 200 & 13074 \\
0.30 & static & 300 & 10081 \\
0.30 & adj & 350 & 9636 \\
\hline 0.35 & deterministic & 200 & infeasible \\
0.35 & static & 350 & 10565 \\
0.35 & adj & 350 & 10565 \\
\hline
\end{tabular}




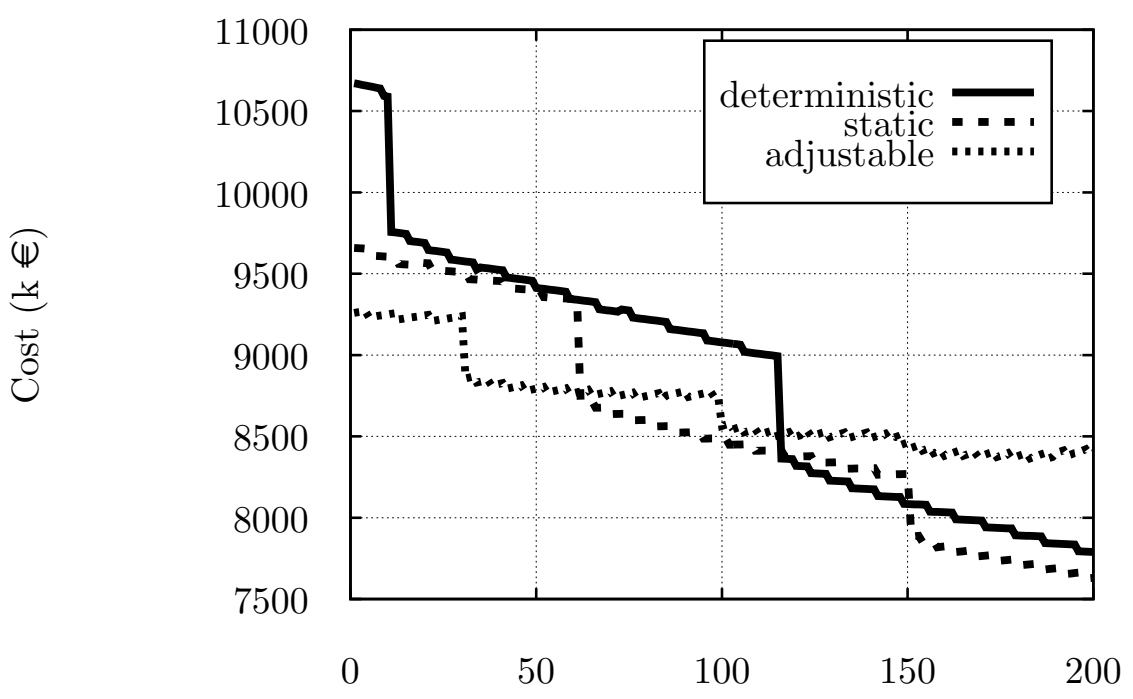

First level realization

Figure 4: Simulations with first-stages imposed for $\Gamma=0.25$ deviation

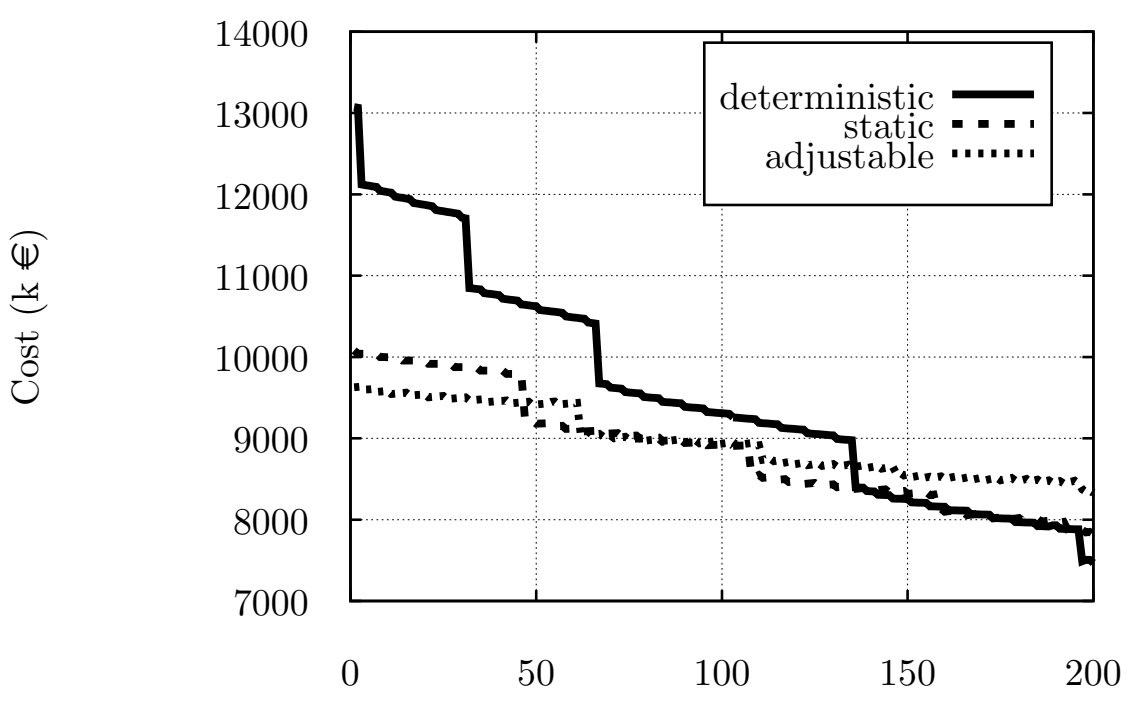

First level realization

Figure 5: Simulations with first-stages imposed for $\Gamma=0.30$ 


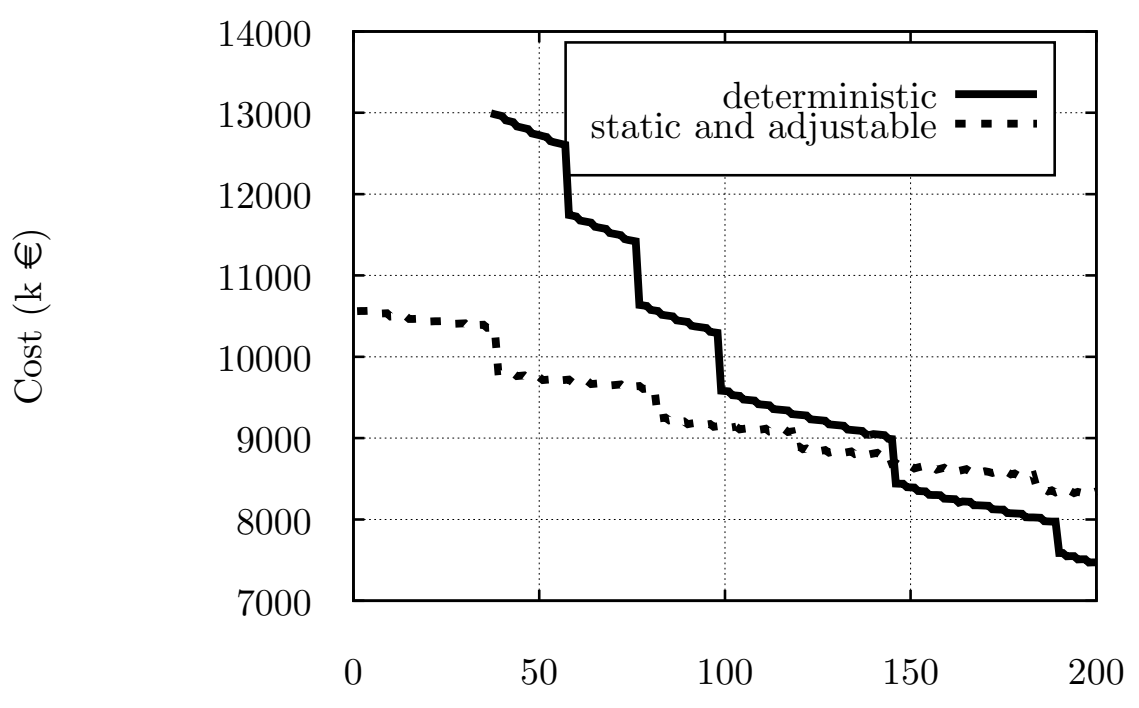

First level realization

Figure 6: Simulations with first-stages imposed for $\Gamma=0.35$

\section{Scalability tests of the formulations}

In this section, we assess the scalability of our different formulations in deterministic and uncertain contexts on realistic instances of different sizes and characteristics. We also evaluate the impact of the proposed RLT cuts. We first describe in Section 5.1 the characteristics of the instances used for these scalability experiments. Our results for deterministic and robust formulations are presented in Section 5.2.

\subsection{Instances parameters}

We test six different sizes (number of sites) for the considered instances: 25, 50, 75, 100, 125 and 150 as well as three density types: Rural, Suburban and Urban. The distinction between these three types of instances are the initial $4 \mathrm{G}$ deployment : $17 \%$ in rural areas, $34 \%$ in suburban areas, $68 \%$ in urban areas.

In all the tests presented here, we take, as in Section $4.2, \underline{Q o E}=0.8$ and $\underline{\alpha}=0.7$. The nominal shifting function values $\tilde{f}_{\sigma, c}$ are also given by Table 3 . For the rural instances, since the initial coverage is $17 \%(<25 \%)$, we add to this table a row for the coverage level "low" (from 0 to $25 \%$ initial coverage) and consider that there is no reaction of the subscribers at this level 
$\left(f_{\sigma, \text { low }}=0, \forall \sigma \in \mathcal{K}\right)$. For assessing our robust models, we use the asymmetric uncertainty set introduced in Section 4, with a deviation $\Gamma=0.2$.

\subsection{Results}

The results obtained on this set of 18 instances are presented in Tables 6-8. The features of the instances are stored in these tables under multicolumn "Instance", with "NS" standing for the number of sites and "den" for the density (Rural "R", Suburban "S" and Urban "U"). The other columns present the results obtained with the following models:

- Deterministic model (multi-column "deterministic"): formulations $\mathcal{M}^{\text {det }}$ and $\mathcal{M}^{\text {det,2p }}$,

- Static model with $\Gamma=0.2$ (multi-column "static"): formulation $\mathcal{M}^{\text {stat }}$,

- Adjustable model with $\widehat{L}=2$ and $\widehat{L}=4$ (multi-column "adjustable"): formulations $\widehat{\mathcal{M}}^{\text {adj,2 }}$ and $\widehat{\mathcal{M}}^{a d j, 4}$. 
Table 6: Root gaps on the set of 18 instances for all deterministic and robust formulations

\begin{tabular}{|c|c|c|c|c|c|c|c|c|c|c|c|}
\hline \multirow{2}{*}{\multicolumn{2}{|c|}{ Instance }} & \multicolumn{10}{|c|}{ Root gap } \\
\hline & & \multicolumn{4}{|c|}{ deterministic } & \multicolumn{2}{|r|}{ static } & \multicolumn{4}{|c|}{ adjustable } \\
\hline $\mathrm{NS}$ & den & $\mathcal{M}^{\text {det }}$ & $\mathcal{M}^{d e t}+R L T$ & $\mathcal{M}^{\text {det }, 2 p}$ & $\mathcal{M}^{d e t, 2 p}+R L T$ & $\mathcal{M}^{\text {stat }}$ & $\mathcal{M}^{\text {stat }}+R L T$ & $\widehat{\mathcal{M}}^{a d j, 2}$ & $\widehat{\mathcal{M}}^{a d j, 2}+R L T$ & $\widehat{\mathcal{M}}^{a d j, 4}$ & $\widehat{\mathcal{M}}^{a d j, 4}+R L T$ \\
\hline \multirow{3}{*}{25} & $\mathrm{R}$ & 16.20 & 16.20 & 5.13 & 2.51 & 4.55 & 4.26 & 4.40 & 4.27 & 4.35 & 4.28 \\
\hline & $\mathrm{S}$ & 27.82 & 20.24 & 125.39 & 3.03 & 125.91 & 5.05 & 125.92 & 5.50 & 126.38 & 4.78 \\
\hline & $\mathrm{U}$ & 32.92 & 22.18 & 156.42 & 3.57 & 144.43 & 6.04 & 144.44 & 6.31 & 144.61 & 6.93 \\
\hline \multirow{3}{*}{50} & $\mathrm{R}$ & 16.12 & 16.12 & 3.86 & 1.55 & 1.59 & 1.37 & 1.46 & 1.39 & 1.42 & 1.39 \\
\hline & S & 28.32 & 21.32 & 108.20 & 3.58 & 107.88 & 5.00 & 108.03 & 4.23 & 108.04 & 4.92 \\
\hline & $\mathrm{U}$ & 32.49 & 22.68 & 154.53 & 5.18 & 141.56 & 6.37 & 141.53 & 6.71 & 141.50 & 8.15 \\
\hline \multirow{3}{*}{75} & $\mathrm{R}$ & 14.77 & 14.77 & 2.44 & 0.66 & 7.19 & 7.07 & 7.13 & 7.08 & 7.11 & 7.08 \\
\hline & $\mathrm{S}$ & 27.06 & 20.37 & 106.64 & 1.99 & 106.90 & 1.86 & 106.64 & 2.44 & 106.32 & 2.75 \\
\hline & $\mathrm{U}$ & 32.87 & 23.61 & 153.94 & 5.49 & 141.10 & 6.76 & 141.02 & 7.11 & 141.75 & 6.98 \\
\hline \multirow{3}{*}{100} & $\mathrm{R}$ & 15.11 & 15.11 & 2.10 & 0.61 & 8.09 & 7.97 & 8.02 & 7.97 & 10.67 & 7.98 \\
\hline & $\mathrm{S}$ & 26.74 & 19.84 & 108.14 & 1.86 & 107.60 & 4.75 & 107.54 & 4.60 & 104.47 & 4.88 \\
\hline & $\mathrm{U}$ & 33.37 & 24.15 & 153.76 & 6.01 & 141.41 & 6.38 & 141.31 & 6.70 & 142.00 & 6.64 \\
\hline \multirow{3}{*}{125} & $\mathrm{R}$ & 15.61 & 15.61 & 2.44 & 1.03 & 1.52 & 1.39 & 1.45 & 1.40 & 36.72 & 17.74 \\
\hline & $\mathrm{S}$ & 27.37 & 20.44 & 107.55 & 2.74 & 107.17 & 4.56 & 107.30 & 4.52 & 103.87 & 6.24 \\
\hline & $\mathrm{U}$ & 33.97 & 24.90 & 132.79 & 5.47 & 121.62 & 6.18 & 122.01 & 4.63 & 118.17 & 6.26 \\
\hline \multirow{3}{*}{150} & $\mathrm{R}$ & 15.87 & 15.87 & 2.52 & 1.07 & 2.64 & 2.51 & 2.58 & 2.52 & 41.79 & 41.44 \\
\hline & $\mathrm{S}$ & 27.07 & 20.45 & 106.17 & 1.96 & 106.39 & 1.97 & 105.61 & 2.52 & 102.89 & 52.99 \\
\hline & $\mathrm{U}$ & 33.61 & 24.36 & 133.44 & 5.10 & 122.16 & 5.55 & 122.10 & 5.84 & 119.15 & 5.78 \\
\hline
\end{tabular}


Table 7: Final gaps/solving times on the set of 18 instances for all deterministic and robust formulations

\begin{tabular}{|c|c|c|c|c|c|c|c|c|c|c|c|}
\hline \multirow{2}{*}{\multicolumn{2}{|c|}{ Instance }} & \multicolumn{10}{|c|}{ Final gap } \\
\hline & & \multicolumn{4}{|c|}{ deterministic } & \multicolumn{2}{|r|}{ static } & \multicolumn{4}{|c|}{ adjustable } \\
\hline NS & den & $\mathcal{M}^{\text {det }}$ & $\mathcal{M}^{\text {det }}+R L T$ & $\mathcal{M}^{d e t, 2 p}$ & $\mathcal{M}^{d e t, 2 p}+R L T$ & $\mathcal{M}^{\text {stat }}$ & $\mathcal{M}^{\text {stat }}+R L T$ & $\widehat{\mathcal{M}}^{a d j, 2}$ & $\widehat{\mathcal{M}}^{a d j, 2}+R L T$ & $\widehat{\mathcal{M}}^{a d j, 4}$ & $\widehat{\mathcal{M}}^{a d j, 4}+R L T$ \\
\hline \multirow{3}{*}{25} & $\mathrm{R}$ & 1 & 1 & 39 & 15 & 37 & 18 & 232 & 80 & 884 & 352 \\
\hline & S & 5 & 7 & 184 & 33 & 149 & 39 & 396 & 180 & 1723 & 920 \\
\hline & $\mathrm{U}$ & 1 & 1 & 22 & 19 & 20 & 5 & 76 & 48 & 264 & 269 \\
\hline \multirow{3}{*}{50} & $\mathrm{R}$ & 15 & 3 & 126 & 66 & 113 & 58 & 901 & 340 & 1565 & 1413 \\
\hline & $\mathrm{S}$ & 41 & 84 & 503 & 227 & 515 & 105 & 4227 & 772 & 4.40 & 3744 \\
\hline & $\mathrm{U}$ & 39 & 23 & 88 & 42 & 78 & 34 & 290 & 306 & 1406 & 433 \\
\hline \multirow{3}{*}{75} & $\mathrm{R}$ & 53 & 11 & 342 & 122 & 194 & 72 & 2949 & 621 & 5398 & 2280 \\
\hline & $\mathrm{S}$ & 209 & 364 & 1950 & 186 & 612 & 112 & 8.81 & 920 & 31.68 & 2855 \\
\hline & $\mathrm{U}$ & 199 & 155 & 203 & 49 & 271 & 78 & 662 & 400 & 3104 & 761 \\
\hline \multirow{3}{*}{100} & $\mathrm{R}$ & 215 & 31 & 520 & 316 & 316 & 116 & 0.16 & 1223 & 9.48 & 4200 \\
\hline & $\mathrm{S}$ & 554 & 472 & 1564 & 376 & 2034 & 547 & 13.09 & 0.99 & 57.33 & 1.02 \\
\hline & $\mathrm{U}$ & 743 & 380 & 396 & 828 & 758 & 109 & 1840 & 1089 & 4696 & 3547 \\
\hline \multirow{3}{*}{125} & $\mathrm{R}$ & 504 & 655 & 711 & 597 & 617 & 220 & 3993 & 5218 & 33.70 & 14.85 \\
\hline & $\mathrm{S}$ & 0.01 & 5409 & 0.01 & 4962 & 0.08 & 419 & 6.71 & 0.98 & 64.72 & 3.83 \\
\hline & $\mathrm{U}$ & 1766 & 735 & 331 & 259 & 334 & 176 & 2410 & 1356 & 28.37 & 2926 \\
\hline \multirow{3}{*}{150} & $\mathrm{R}$ & 2207 & 1454 & 1010 & 1210 & 975 & 261 & 0.17 & 3998 & INF & INF \\
\hline & $\mathrm{S}$ & 5654 & 3456 & 6361 & 1061 & 0.11 & 422 & 45.49 & 2510 & 70.23 & INF \\
\hline & $\mathrm{U}$ & 3310 & 2514 & 821 & 424 & 569 & 182 & 1.59 & 1086 & 20.49 & 6891 \\
\hline
\end{tabular}


Table 8: Best solution found on the set of 18 instances for all deterministic and robust formulations

\begin{tabular}{|c|c|c|c|c|c|c|c|c|c|c|c|}
\hline \multirow{2}{*}{\multicolumn{2}{|c|}{ Instance }} & \multicolumn{10}{|c|}{ Solution Value } \\
\hline & & \multicolumn{4}{|c|}{ deterministic } & \multicolumn{2}{|r|}{ static } & \multicolumn{4}{|c|}{ adjustable } \\
\hline & den & $\mathcal{M}^{\text {det }}$ & $\mathcal{M}^{\text {det }}+R L T$ & $\mathcal{M}^{\text {det }, 2 p}$ & $\mathcal{M}^{d e t, 2 p}+R L T$ & $\mathcal{M}^{\text {stat }}$ & $\mathcal{M}^{\text {stat }}+R L T$ & $\widehat{\mathcal{M}}^{a d j, 2}$ & $\widehat{\mathcal{M}}^{a d j, 2}+R L T$ & $\widehat{\mathcal{M}}^{a d j, 4}$ & $\widehat{\mathcal{M}}^{a d j, 4}+R L T$ \\
\hline \multirow{3}{*}{25} & $\mathrm{R}$ & 1990 & 1990 & 1990 & 1990 & 3051 & 3051 & 3051 & 3051 & 3051 & 3051 \\
\hline & $\mathrm{S}$ & 1656 & 1656 & 1656 & 1656 & 2178 & 2178 & 2178 & 2178 & 2143 & 2143 \\
\hline & $\mathrm{U}$ & 1365 & 1365 & 1365 & 1365 & 1939 & 1939 & 1939 & 1939 & 1932 & 1932 \\
\hline \multirow{3}{*}{50} & $\mathrm{R}$ & 3884 & 3884 & 3884 & 3884 & 5872 & 5872 & 5872 & 5872 & 5872 & 5872 \\
\hline & $\mathrm{S}$ & 3446 & 3446 & 3446 & 3446 & 4560 & 4560 & 4498 & 4498 & 4501 & 4498 \\
\hline & $\mathrm{U}$ & 2832 & 2832 & 2832 & 2832 & 4027 & 4027 & 4027 & 4027 & 4027 & 4027 \\
\hline \multirow{3}{*}{75} & $\mathrm{R}$ & 5858 & 5858 & 5858 & 5858 & 9616 & 9616 & 9616 & 9616 & 9616 & 9616 \\
\hline & $\mathrm{S}$ & 4852 & 4852 & 4852 & 4852 & 6291 & 6291 & 6529 & 6291 & 6854 & 6273 \\
\hline & $\mathrm{U}$ & 4160 & 4160 & 4160 & 4160 & 5916 & 5916 & 5916 & 5916 & 5806 & 5806 \\
\hline \multirow{3}{*}{100} & $\mathrm{R}$ & 7799 & 7799 & 7799 & 7799 & 12846 & 12846 & 12846 & 12846 & 13231 & 12846 \\
\hline & $\mathrm{S}$ & 6699 & 6699 & 6699 & 6699 & 9015 & 9015 & 9089 & 8950 & 15320 & 8913 \\
\hline & $\mathrm{U}$ & 5329 & 5329 & 5329 & 5329 & 7502 & 7502 & 7502 & 7502 & 7367 & 7367 \\
\hline \multirow{3}{*}{125} & $\mathrm{R}$ & 9588 & 9588 & 9588 & 9588 & 14531 & 14531 & 14531 & 14531 & 22633 & 17416 \\
\hline & $\mathrm{S}$ & 8435 & 8435 & 8435 & 8435 & 11356 & 11202 & 11137 & 11137 & 20965 & 11260 \\
\hline & $\mathrm{U}$ & 6315 & 6315 & 6315 & 6315 & 8893 & 8893 & 8720 & 8720 & 10552 & 8720 \\
\hline \multirow{3}{*}{150} & $\mathrm{R}$ & 11226 & 11226 & 11226 & 11226 & 17022 & 17022 & 17022 & 17022 & 28496 & 28335 \\
\hline & $\mathrm{S}$ & 9467 & 9467 & 9467 & 9467 & 12337 & 12296 & 13996 & 12296 & 27047 & 25330 \\
\hline & $\mathrm{U}$ & 7662 & 7662 & 7662 & 7662 & 10778 & 10778 & 10778 & 10778 & 12424 & 10592 \\
\hline
\end{tabular}


For formulation $\mathcal{M}^{\text {det }}$, we provide results without and with (labeled by "+RLT") linearization cut from Cambier et al. (2020). For the other formulations, we provide results without and with (labeled by "+RLT") linearization cuts (40)-(46). For each formulation, we provide three indicators: the root gap, defined as the gap between the best solution found by the solver and the value of the continuous relaxation; the final gap, defined as the gap between the best solution found by the solver and the best upper bound found; and the value of the best solution found. When the final gap is closed, we provide instead the solving time value. The results for the root gap are stored in Table 6. The ones for the solving time/final gap are provided in Table 7. A bold value in this table means that the solver has not closed the gap within the two hours time limit (strictly positive gap). The best solutions found are presented in Table 8. A bold value in this table indicates the best robust solution found among all the formulations.

In the remainder of this section, we discuss these results. Deterministic formulations are discussed in Section 5.2.1 while robust formulations are discussed in Section 5.2.2.

\subsubsection{Results for deterministic formulations}

We first analyze the results obtained for the deterministic formulation. We observe in Table 7 that CPLEX solver finds the optimal solution within the time-limit for both the MILP formulation $\mathcal{M}^{\text {det }}$ and the ILP reformulation $\mathcal{M}^{\text {det,2p }}$ on all instances. We observe in the same table, that for both formulations, adding the cuts reduces the solving time of most instances, with a reduction by a factor 10 (186 seconds instead of 1950) for $\mathcal{M}^{\text {det,2p }}$ on the 75 sites suburban instance. When

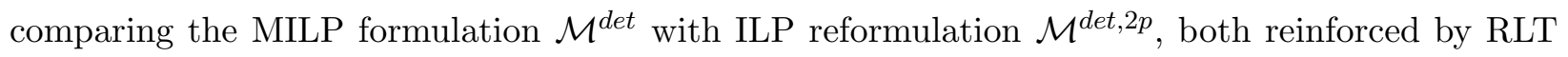
cuts, we see that CPLEX solver converges faster when $\mathcal{M}^{\text {det, } 2 p}$ is used on all six large instances of 125 and 150 sites. For the urban instance of 150 sites, the solving time is divided by 5 (424 seconds instead of 2514). However, these observations do not apply on smaller instances : for 9 of the 12 instances from 25 to 100 sites instances, using reinforced $\mathcal{M}^{\text {det }}$ provides faster solving times than using reinforced $\mathcal{M}^{\text {det,2p}}$.

Regarding the root gap, we observe from results in Table 6 that the cuts always improve the value of the continuous relaxation. In the initial MILP formulation $\mathcal{M}^{\text {det }}$, adding the cuts reduces indeed the average root gap by $7 \%$ in suburban instances, $9 \%$ in urban instances, and has no effect in rural instances. Regarding the ILP formulation $\mathcal{M}^{d e t, 2 p}$ without the cuts, the root gap is above $100 \%$ for suburban and urban instances, while it stands between 2 and $5 \%$ for rural instances. This 
behavior in the rural instances can be explained by the absence of subsidies in the first period: since the coverage was initially at level "low", we know that the subscribers do not react in the first period, and therefore there is no need to propose any subsidy. The bad performances of the continuous relaxation in both suburban and urban instances come hence from the linearization of first period decisions. The reinforcement of such linearizations by the RLT cuts proves to be particularly valuable, as it enables to contain the root gaps under $6 \%$ for all types of instances (5 times better than the MILP without cuts, 4 times better than the MILP with cuts, 25 times better than the ILP without cuts).

In Table 8, we observe that for each instance, the deterministic formulations are equivalent in terms of solution obtained, since they provide the optimal solution.

\subsubsection{Results for robust formulations}

We now turn our attention for the results obtained by the robust formulation. We observe the same impact of using the cuts on the root gap than in the deterministic case: for example, in the adjustable formulation with $\widehat{L}=2$, the maximal root gap is around $144 \%$ while it is only $8 \%$ for the reinforced formulation.

For the static formulation, we observe in Table 7 that the formulation without reinforcement proves the optimality of the solution for all instances except the suburban ones of 125 and 150 sites, which have a very low final gap of $0.08 \%$ (solution value of 11356 ) and $0.11 \%$ (solution value of 12337) respectively. When cuts are added, the optimal solutions of respective value 11202 and 12296 are found and proven to be optimal in two hours by CPLEX.

Regarding the adjustable formulations, we observe in Table 7 that the increase of the instances size makes the formulation more difficult. CPLEX does not converge in two hours on 7 instances

for the model with $\widehat{L}=2$ and on 10 instances for the model where $\widehat{L}=4$. Here again, the RLT cuts prove themselves to be particularly valuable, as they reduce these numbers of instances to 2 for the model with $\widehat{L}=2$ and to 5 for the model with $\widehat{L}=4$. Besides, when the solver does not converge, the gap has been considerably reduced, as it can be seen for the 125 suburban instances: $0.98 \%$ with cuts compared to $6.71 \%$ without when $\widehat{L}=2$ and $3.83 \%$ with cuts compared to $64.72 \%$ without when $\widehat{L}=4$.

Finally, Table 8 gives an idea of the robust formulation that provides the best solution. From 
the results in this table, we observe that the reinforced adjustable formulation with $\widehat{L}=4$ provides the best solution for all instances except the rural and suburban instances of 125 and 150 sites. On 7 instances, the solution found using the reinforced adjustable formulation with $\widehat{L}=4$ is strictly better than the solution found using the reinforced adjustable formulation with $\widehat{L}=2$. For the rural and suburban instances of 125 and 150 sites, the reinforced adjustable formulation with $\widehat{L}=2$ provides the best solution.

\section{Conclusion}

In this paper, we have introduced and strengthened a new formulation for the two periods and two generations mobile master plan problem using only integer variables. Assuming that the discrete shifting function can take any value in a given polytope, and optimizing against the worst-case outcome, we have proposed a robust counterpart for the problem. We have handled integer recourse variables by partitioning the uncertainty set while the non-linear dependencies on the uncertain parameters have been tackled by a careful analysis of dominating scenarios. Numerical experiments have been performed for static and adjustable robust frameworks on a 100 sites instance, with an uncertainty set controlling variation around the nominal value and parametrized by an amplitude $\Gamma$. All formulations have been assessed from a scalability point-of-view and our results have enlightened the benefit from the cuts in improving the formulations. The two formulations proposed for the deterministic model are scalable. The new formulation $\mathcal{M}^{\text {det,2p }}$ reduces the solving time for the largest instances. Regarding the robust results, as expected, the adjustable model with a refined partition $(\widehat{L}=4)$ finds solutions of lower cost for most instances, but for the largest instances, the static model or the adjustable model with $\widehat{L}=2$ should be privileged due to better scalability. Our results on an operational case-study have illustrated the potential economical relevance of the static and adjustable first-stage decisions over the deterministic one. These decisions can lead to saving costs as high as $30 \%$ of the total costs in the case $\Gamma=0.3$, while the deterministic solution can become infeasible for $\Gamma=0.35$. Our results have also underlined the possible impact of the quality of experience threshold, which is responsible for the higher subsidies decisions and the higher costs it involves.

This work has paved the way for two numerical extensions. First, it would be interesting to assess rolling horizon heuristics to handle problems with more than two periods. Second, the operator 
could use these models on specific and relevant use-cases to assess more precisely the situations in which robust (static or adjustable) solutions should be preferred over the deterministic ones.

\section{References}

Bass, F.M., 1969. A new product growth for model consumer durables. Management Science 15, 215-227. URL: https://doi.org/10.1287/mnsc.15.5.215, doi:10.1287/mnsc.15.5.215.

Bass, F.M., 2004. Comments on "a new product growth for model consumer durables the bass model". Management Science 50, 1833-1840. URL: https://doi.org/10.1287/mnsc.1040.0300, doi:10.1287/mnsc.1040.0300, arXiv:https://doi.org/10.1287/mnsc.1040.0300.

Ben-Ameur, W., Zotkiewicz, M., 2011. Robust routing and optimal partitioning of a traffic demand polytope. Int. Trans. Oper. Res. 18, 307-333. URL: https://doi.org/10.1111/j.1475-3995.2010.00764.x, doi:10.1111/j.14753995.2010.00764.x.

Ben-Tal, A., El Ghaoui, L., Nemirovski, A., 2009. Robust optimization. volume 28. Princeton University Press.

Ben-Tal, A., den Hertog, D., Vial, J., 2015. Deriving robust counterparts of nonlinear uncertain inequalities. Mathematical Programming 149, 265-299. URL: https://doi.org/10.1007/s10107-014-0750-8, doi:10.1007/s10107-014-0750-8.

Ben-Tal, A., Nemirovski, A., Roos, C., 2002. Robust solutions of uncertain quadratic and conic-quadratic problems. SIAM Journal on Optimization 13, 535-560. URL: https://doi.org/10.1137/S1052623401392354, doi:10.1137/S1052623401392354.

Bertsimas, D., Caramanis, C., 2010. Finite adaptability in multistage linear optimization. IEEE Trans. Automat. Contr. 55, 2751-2766. URL: https://doi.org/10.1109/TAC.2010.2049764, doi:10.1109/TAC.2010.2049764.

Bertsimas, D., Dunning, I., 2016. Multistage robust mixed-integer optimization with adaptive partitions. Operations Research 64,980-998. URL: https://doi.org/10.1287/opre.2016.1515, doi:10.1287/opre.2016.1515. 
Bertsimas, D., Dunning, I., Lubin, M., 2015. Reformulation versus cutting-planes for robust optimization. Computational Management Science 13. doi:10.1007/s10287-015-0236-z.

Bertsimas, D., Georghiou, A., 2018. Binary decision rules for multistage adaptive mixed-integer optimization. Mathematical Programming 167, 395-433. URL: https://doi .org/10.1007/s10107-017-1135-6, doi:10.1007/s10107-017-1135-6.

Buchheim, C., Kurtz, J., 2018. Robust combinatorial optimization under convex and discrete cost uncertainty. EURO Journal on Computational Optimization 6, 211-238. URL: https://doi .org/10.1007/s13675-018-0103-0, doi:10.1007/s13675-018-0103-0.

Cambier, A., Chardy, M., Figueiredo, R., Ouorou, A., Poss, M., 2020. Optimizing the investments in mobile networks and subscriber migrations for a telecommunication operator. Networks n/a. URL: https://onlinelibrary.wiley.com/doi/abs/10.1002/net.21970, doi:https://doi.org/10.1002/net.21970, arXiv:https://onlinelibrary.wiley.com/doi/pdf/10.1002/net.21

CISCO, 2017. Cisco visual networking index: Global mobile data traffic forecast update, 2016-2021 white paper. URL: https://www.cisco.com/c/en/us/solutions/collateral/service-provider/visual-networking-index(Accessed 10/30/2018).

Delage, E., Iancu, D., 2015. Robust multistage decision making. informs tutorials in operations research. TutORials in Operations Research .

Dunning, I., Huchette, J., Lubin, M., 2017. Jump: A modeling language for mathematical optimization. SIAM Review 59, 295-320. URL: https://doi.org/10.1137/15M1020575, doi:10.1137/15M1020575, arXiv:https://doi.org/10.1137/15M1020575.

Fortet, 1960. Boole algebra and its application to operation research. Trabajos de Estadistica, $111-118$.

Gabrel, V., Murat, C., Thiele, A., 2014. Recent advances in robust optimization: An overview. European Journal of Operational Research 235, 471-483. doi:10.1016/j.ejor.2013.09.036.

Norton, J.A., Bass, F.M., 1987. A diffusion theory model of adoption and substitution for successive generations of high-technology products. Management Science 33, 
1069-1086. URL: https://doi.org/10.1287/mnsc.33.9.1069, doi:10.1287/mnsc.33.9.1069, arXiv:https://doi.org/10.1287/mnsc.33.9.1069.

Orange, 2018. Mobile network worlwide activity (orange group webpage). URL: https://www.orange.com/en/Group/Activities/Networks/Folder/Mobile-network (Accessed 10/30/2018).

Pessoa, A.A., Poss, M., 2015. Robust network design with uncertain outsourcing cost. INFORMS Journal on Computing 27, 507-524. URL: https://doi.org/10.1287/ijoc.2015.0643, doi:10.1287/ijoc.2015.0643.

Postek, K., den Hertog, D., 2016. Multistage adjustable robust mixed-integer optimization via iterative splitting of the uncertainty set. INFORMS Journal on Computing 28, 553-574. URL: https://doi .org/10.1287/ijoc.2016.0696, doi:10.1287/ijoc.2016.0696.

Sherali, H., Adams, W., 1998. A Reformulation-Linearization Technique for Solving Discrete and Continuous Nonconvex Problems. Nonconvex Optimization and Its Applications, Springer US. URL: https://books.google.fr/books?id=sPzzL4VvWqsC.

İhsan Yanıkoğlu, Gorissen, B.L., den Hertog, D., 2019. A survey of adjustable robust optimization. European Journal of Operational Research 277, 799 - 813. URL: $\quad$ http://www.sciencedirect.com/science/article/pii/S0377221718307264, doi:https://doi.org/10.1016/j.ejor.2018.08.031. 